\title{
Stage-Structured Population Systems with Temporally Periodic Delay
}

\section{Xiaotiān $W_{u^{\dagger}}$, Felicia Maria G. Magpantay $y^{\ddagger}$ Jianhong $\mathbf{W u}^{\S *}$, Xingfu Zou In honour of Professor Messoud Efendiev on his 60th Birthday}

For some ectotherms such as Ixodes scapularis, a vector of Lyme disease, changes in temperature is believed to affect the interstadial development time and hence give rise to a time-periodic delay due to seasonality in the population dynamics described by a stage-structured population growth model. Here, we develop a formulation linking the chronological delay with multiple stage-specific interstadial delays. We also present a definition for the basic reproductive ratio for such a system, develop a simple algorithm to compute it, and show that the results regarding the stability of the zero solution are consistent with those from computing the dominant Floquet multiplier. Numerical simulations also show that the threshold value for the population persistence or extinction depends not only on the mean but also on the amplitude and phase of the periodic development delays. Copyright (c) 2009 John Wiley \& Sons, Ltd.

Keywords: Temperature change; stage-structured; basic reproductive ratio; periodic time delay; dominant Floquet multiplier

\section{Introduction}

For some ectothermic insects such as deer ticks (Ixodes scapularis), the interstadial development time is temperature-dependent, with higher temperatures typically resulting in shorter development times [22, 23, 25, 32, 33]. Motivated by this physiological characteristics of such populations, we formulate an appropriate mechanistic model that describes the evolution of the population in each biologically distinctive stage under seasonally varying periodic environment from general structured population dynamics models [37]. To parametrize the model and to link model simulations to observed data, we formulate the model as a system of delay differential equations for which each state variable corresponds to the population size at a given biological stage. This formulation permits rigorous mathematical analysis and calculation of key threshold indices that determine if a population will survive or go extinct. This, as will be shown, requires the exploration of the iterative relationship between development times, and the minimum and maximum ages of the population in each state.

A systematic approach has been developed in the pioneering work of Nisbet and Gurney [21] to derive population models for insects with dynamically varying instar duration. Here we develop a formulation to link the insect chronological age with the insect stage-specific age which corresponds to the physiological mass in the work of Nisbet and Gurney [21], and Metz and Diekmann [20]. We obtain a system of delay differential equations with time-periodic lags which are explicitly given using

This is the author manuscript accepted for pubnication and has undergone fun peer reviev but has

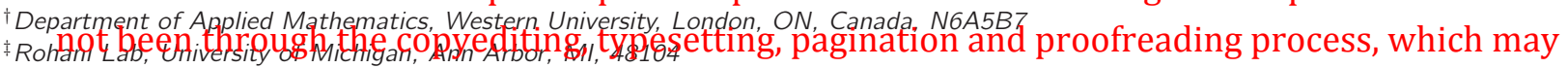

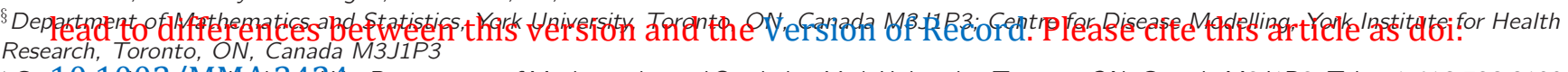

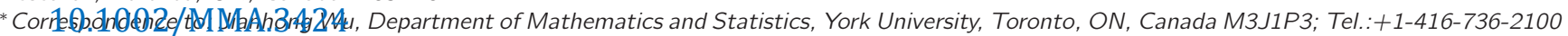
ext.33116; email: wujh@mathstat.yorku.ca 
a specific chronological age and stage-specific age relationship (Eq. (9)). This facilitates some qualitative study of the model system, in particular, for the formal definition and some computation of a basic reproductive ratio, denoted by $\mathcal{R}_{0}$.

This ratio, one of the most important and useful quantities in the field of ecology and infectious disease epidemiology, has been considered and studied extensively in deterministic models. In epidemiology, the value of $\mathcal{R}_{0}$ characterizes the potential growth of the population of infected individuals after one infected individual is introduced into a completely susceptible population. In general, $\mathcal{R}_{0}>1\left(\mathcal{R}_{0}<1\right)$ indicates that the size of infected population will increase (decrease) and the disease will become endemic (die out) (see $[1,11,12,29,35]$ for detailed definitions and some applications). In ecology, $\mathcal{R}_{0}$ accounts for the average number of reproductive offspring produced by a single reproductive member in its life span. As such, the population will grow if $\mathcal{R}_{0}>1$, while the population cannot establish in the ecosystem if $\mathcal{R}_{0}<1[18,38,39]$. The value of $\mathcal{R}_{0}$ plays a threshold role in determining the stability of the corresponding linearized system at the infection-free or population-extinction steady state. A natural way to define $\mathcal{R}_{0}$ for a model system is to consider the linearization of the model system at this steady state. The matrix describing the linear system is broken down into either the $F-V$ (or $T+\Sigma$ ) decomposition [11, 12, 29] in an autonomous ODE system, or the $F(t)-V(t)$ decomposition in a periodic ODE system [35]. Here $F$ (or $T, F(t))$ is the transmission matrix in epidemiology (or the new-borne matrix in ecology) describing the new infections or new-borne females, and $V$ (or $-\Sigma, V(t)$ ) is the progressive matrix describing the change of each state due to death, development and removal. For an autonumous ODE system, $\mathcal{R}_{0}$ is defined as the spectral radius of the so-called the next generation matrix, $F V^{-1}$ (or $-T \Sigma^{-1}$ ) and the value is computed as the dominant eigenvalue of $F V^{-1}$. For a periodic nonlinear ODE system, $\mathcal{R}_{0}$ is defined as the spectral radius of a next generation operator $\mathcal{L}$ constructed from the matrices $F(t)$ and $V(t)[2,35]$. There are some numerical algorithms for calculating $\mathcal{R}_{0}[3,35]$ and there have already been quite a few applications using periodic ordinary differential equations $[3,6,14,16,18,28,30,39]$ and delay differential equations with discrete and time-constant delay [4, 5]. There is also a way to define this threshold value for stochastic differential equations $[10,36,40]$, which determines the probability of a primary outbreak. Wangombe et al. [36] has also studied this for a stochastic model of tick-borne diseases.

Here, we use some of these methodologies to formally define the basic reproductive ratio of the kind of system of delay differential equations with periodic delay we are considering, and to propose and validate a numerical algorithm for computing $\mathcal{R}_{0}$. More specifically, we follow the idea of [2] for a definition of the basic reproductive ratio in a periodic environment, using the renewal equation satisfied by the birth rate $y(t)[2,17,34,35]$ :

$$
y(t)=\int_{0}^{\infty} \mathcal{K}(t, r) y(t-r) d r
$$

where the kernel $\mathcal{K}(t, r)$ is $\omega$-periodic with respect to $t$ and the basic reproductive ratio was defined to be the spectral radius of the next generation integral operator

$$
\mathcal{L}: u(t) \mapsto \int_{0}^{\infty} \mathcal{K}(t, r) u(t-r) d x
$$

acting on the function space consisting of $\omega$-periodic continuous functions.

This paper is organized as follows: In Section 2, we derive a stage-structured model of delay differential equations with periodic delay and verify the well-posedness of the system. Section 3 gives the detailed derivation of the basic reproductive ratio of the model and proposes a numerical algorithm for calculating $\mathcal{R}_{0}$. In Section 4, we present numerical simulations to illustrate the algorithm and some insights about the dependence of $\mathcal{R}_{0}$ on model parameters. We also compare the proposed $\mathcal{R}_{0}$ values with those of the dominant Floquet multiplier. We show that our results are consistent with the those obtained by using the existing algorithms for the dominant Floquet multiplier. A brief discussion is given in the last section.

\section{Model Formulation}

Here, we develop a general dynamic population model where the interstadial development time of the population from one life stage to the next has considerable variation due to temperature change. Due to seasonality, these development times should really be time-dependent, and a periodic function may offer a reasonable approximation. In our formulation, a key assumption is that the transition times between consecutive stages lead to periodic delays. Here, we subdivide the life cycle of a given 
population into $n$ stages and assume that each stage embodies a specific point of the life of the individual. We denote the size of subpopulations at the $j^{\text {th }}$ stage by $x_{j}(2 \leq j \leq n)$. These stages are in order of increasing maturity (e.g. egg, various larvae, nymphs and adult stages) by $x_{j}$. We reserve $x_{1}$ to represent the size of the mature subpopulation who are able to produce offspring (egg-laying females). We also assume that $\delta$ is the fixed sex ratio for such population.

\subsection{Overall assumptions}

In order to appropriately formulate the mathematical model, we make the following assumptions:

(H1) Let $\tau_{i}(t)$ represent the time period that a newly developed tick state $x_{i+1}$ at time $t$ is evolved from the previous tick state $x_{i}$ at time $t-\tau_{i}(t)$. Assume that temperature varies periodically with period $\omega=365$ days and that $\tau_{i}(t)(i=2, \cdots, n)$ is a non-negative periodic function of $t$ with the same period. We also require that $1-\tau_{i}^{\prime}(t) \geq 0$, which excludes the possibility of the $i^{\text {th }}$ stage of the tick going back to the previous $(i-1)^{\text {th }}$ stage except by birth.

(H2) There is a good reason to assume that stage-wise mortality rate depends on the size of subpopulation at that particular stage due to host grooming behavior or host resistance [23]. Thus we assume that the mortality rate $\mu(t, a)$ at time $t$ and age $a$ is density-dependent and age-dependent, given by the following piecewise function:

$$
\mu(t, a)= \begin{cases}\mu_{1}\left(x_{1}(t)\right), & a \in\left[A_{n}(t), \infty\right), \\ \mu_{i}\left(x_{i}(t)\right), & a \in\left[A_{i-1}(t), A_{i}(t)\right], \quad i=2, \cdots, n,\end{cases}
$$

where $\overline{A_{i-1}(t)}$ and $A_{i}(t)$ are the time-dependent minimum and maximum ages of those individuals who are developing within the specific $i^{\text {th }}$ stage (this will be defined later), and $\mu_{i}$ is a non-decreasing function with $\mu_{i}(0)>0$.

(H3) The birth function of eggs is given by the Ricker function

$$
0\left(x_{1}(t)\right)=p x_{1}(t) e^{-s_{T} x_{1}(t)},
$$

where $p$ is the maximal number of eggs that an egg-laying female can lay per unit time and $s_{T}$ measures the strength of density-dependence. The assumption reflects the ecological consideration that the reproduction is linear in $x_{1}$ only for small densities, decreases as a consequence of intraspecific competition, and then drops significantly at very large densities due to the available resources being utilized by the adults only for their own physiological maintenance.

\subsection{Model derivation}

We now introduce the population chronological age variable $a$ and denote by $\rho(t, a)$ the density of the female population at time $t$ and age a. Following the standard argument for population dynamics with age structure [37], we start with

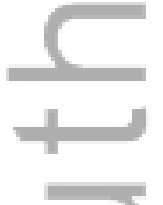

$$
\left\{\begin{array}{l}
\left(\frac{\partial}{\partial t}+\frac{\partial}{\partial a}\right) \rho(t, a)=-\mu(t, a) \rho(t, a) \\
\rho(0, a)=\phi(a), a \geq 0 \\
\rho(t, 0)=b\left(x_{1}(t)\right), \quad t \geq 0
\end{array}\right.
$$

where $\phi(a)$ is the initial age distribution of the population. Integrating (5) along characteristics yields

$$
\rho(t, a)= \begin{cases}\rho(0, a-t) e^{-\int_{0}^{t} \mu(r, a-t+r) d r}, & 0 \leq t \leq a, \\ \rho(t-a, 0) e^{-\int_{0}^{a} \mu(t-a+r, r) d r}, & a<t .\end{cases}
$$

In order to evaluate the rate of change of the specific stage $x_{i}$ at time $t$, we introduce a new variable $\rho_{i}\left(t, a_{i}\right)$ for the density of the female population in the $i^{\text {th }}$ stage at time $t$ and stage-specific age $a_{i}$. In other words, $a_{i}$ is the stage-specific age and $a$ is the population chronological age. Therefore, the total size of female individuals in the $i^{\text {th }}$-stage at time $t\left(x_{i}(t)\right)$ is given by linking the stage-specific age $\left(a_{i}\right)$ and chronological age $(a)$ as follows:

$$
\left\{\begin{array}{l}
x_{1}(t)=\int_{0}^{\infty} \rho_{1}\left(t, a_{1}\right) d a_{1}=\int_{A_{n}(t)}^{\infty} \delta \rho(t, a) d a \\
x_{i}(t)=\int_{0}^{\tau_{i}(t)} \rho_{i}\left(t, a_{i}\right) d a_{i}=\int_{A_{i-1}(t)}^{A_{i}(t)} \rho(t, a) d a, i=2, \cdots, n .
\end{array}\right.
$$


Recall that $A_{i-1}(t)$ and $A_{i}(t)$ are the minimum and maximum ages of those individuals who are developing in the specific $i^{t h}$ stage.

To proceed, we need to know the relationship between chronological age a and stage-specific age $a_{i}$ at time $t$. Note that the population density $\rho(t, a)$ at time $t$ and age $a$ is developed from the density of the population $\rho(t-a, 0)$ at time $t-a$ and age 0 . We depict this as

$$
\rho(t-a, 0) \longrightarrow \rho(t, a)
$$

It is obvious that $a=a_{2}, A_{1}(t)=0$ and $A_{2}(t)=\tau_{2}(t)$. Now $\rho_{3}\left(t, a_{3}\right)$ are developed from themselves at time $t-a_{3}$ and age zero, while $\rho_{3}\left(t-a_{3}, 0\right)$ are developed from the population at time $t-a_{3}-\tau_{2}\left(t-a_{3}\right)$ with the population chronological age zero. Hence,

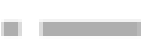

$$
\rho\left(t-a_{3}-\tau_{2}\left(t-a_{3}\right), 0\right) \longrightarrow \rho_{3}\left(t-a_{3}, 0\right) \longrightarrow \rho_{3}\left(t, a_{3}\right) .
$$

Therefore, the stage-specific age $a_{3}$ and the chronological age a are related by

$$
t-a_{3}-\tau_{2}\left(t-a_{3}\right)=t-a
$$

In particular, $a_{3}=0$ is equivalent to $a=\tau_{2}(t)\left(=A_{2}(t)\right)$ and $a_{3}=\tau_{3}(t)$ is the equal of $a=\tau_{3}(t)+\tau_{2}\left(t-\tau_{3}(t)\right)$. Then we obtain

$$
A_{3}(t)=\tau_{3}(t)+\tau_{2}\left(t-\tau_{3}(t)\right) .
$$

Similarly, for each $i=2, \cdots, n$, we obtain an expression determining the time-dependent minimum and maximum age of the population in each specific stage:

$$
A_{i}(t)=\sum_{j=2}^{i} \tau_{j}\left(t-\sum_{k=j+1}^{i} \tau_{k}\left(t-\sum_{l=k+1}^{i} \tau_{l}\left(t-\cdots \tau_{i-1}\left(t-\tau_{i}(t)\right)\right)\right)\right) .
$$

In order to obtain the equation for $x_{i}^{\prime}(t)$, we differentiate $(7)$ along with the assumption $(\mathbf{H} 2)$ and this gives rise to

$$
\begin{aligned}
x_{1}^{\prime}(t) & =\delta\left\{\int_{A_{n}(t)}^{\infty}\left\{\left(\partial_{t}+\partial_{a}\right) \rho(t, a)-\partial_{a} \rho(t, a)\right\} d a-\rho\left(t, A_{n}(t)\right) A_{n}^{\prime}(t)\right\} \\
& =\delta\left\{-\rho(t, \infty)+\rho\left(t, A_{n}(t)\right)-\int_{A_{n}(t)}^{\infty} \mu(t, a) \rho(t, a) d a-\rho\left(t, A_{n}(t)\right) A_{n}^{\prime}(t)\right\} \\
& =\delta \rho\left(t, A_{n}(t)\right)\left(1-A_{n}^{\prime}(t)\right)-\int_{A_{n}(t)}^{\infty} \mu(t, a) \delta \rho(t, a) d a \\
& =\delta \rho\left(t, A_{n}(t)\right)\left(1-A_{n}^{\prime}(t)\right)-\mu_{1}\left(x_{1}(t)\right) x_{1}(t)
\end{aligned}
$$

where we have made the biologically realistic assumption $\rho(t, \infty)=0$.

For $i=2, \cdots, n$, we obtain

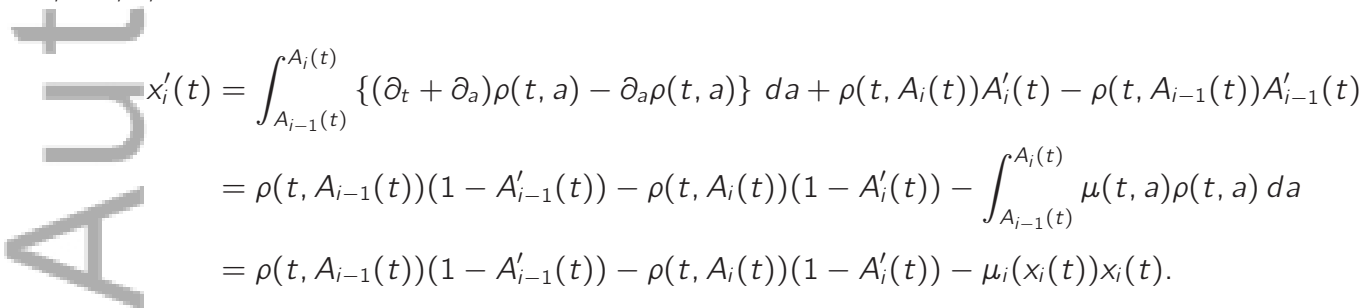


To eventually obtain a closed system for $\left(x_{1}(t), \cdots, x_{n}(t)\right)$, we need to evaluate $\rho\left(t, A_{i}(t)\right)$. This can be done by the method of integration along characteristics. Set $t=t_{0}+s, a=a_{0}+s$, and $V(s)=\rho\left(t_{0}+s, a_{0}+s\right)$. Then

$$
\begin{aligned}
\frac{d V(s)}{d s} & =\left.\left(\frac{\partial}{\partial t} \rho(t, a)+\frac{\partial}{\partial a} \rho(t, a)\right)\right|_{\substack{t=t_{0}+s ; \\
a=a_{0}+s}} \\
& =-\left.\mu\left(t_{0}+s, a_{0}+s\right) \rho(t, a)\right|_{\substack{t=t_{0}+s ; \\
a=a_{0}+s}} \\
& =-\mu\left(t_{0}+s, a_{0}+s\right) V(s) .
\end{aligned}
$$

Note that (12) is a linear first-order ordinary differential equation, we easily obtain

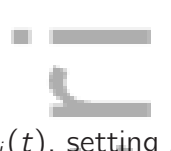

$$
V\left(s_{2}\right)=V\left(s_{1}\right) e^{-\int_{s_{1}}^{s_{2}} \mu\left(t_{0}+r, a_{0}+r\right) d r}
$$

For $t>A_{i}(t)$, setting $s_{2}=A_{i}(t), s_{1}=0, t_{0}=t-A_{i}(t)$, and $a_{0}=0$, we have

$$
V\left(A_{i}(t)\right)=\rho\left(t, A_{i}(t)\right)=\rho\left(t-A_{i}(t), 0\right) e^{-\int_{0}^{A_{i}(t)} \mu\left(t-A_{i}(t)+r, r\right) d r}
$$

With some straightforward calculations, we obtain

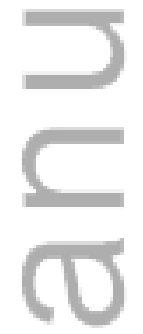

$$
\begin{aligned}
\rho\left(t, A_{2}(t)\right) & =\rho\left(t-A_{2}(t), 0\right) e^{-\int_{0}^{A_{2}(t)} \mu\left(t-A_{2}(t)+r, r\right) d r} \\
& =\rho\left(t-A_{2}(t), 0\right) e^{-\int_{0}^{A_{2}(t)} \mu_{2}\left(x_{2}\left(t-A_{2}(t)+r\right)\right) d r} \\
& =\rho\left(t-A_{2}(t), 0\right) e^{-\int_{t-A_{2}(t)}^{t} \mu_{2}\left(x_{2}(r)\right) d r} \\
& :=\rho\left(t-A_{2}(t), 0\right) \alpha_{2}\left(t, t-A_{2}(t)\right)
\end{aligned}
$$

and

$$
\begin{aligned}
\rho\left(t, A_{3}(t)\right) & =\rho\left(t-\tau_{3}(t), A_{3}(t)-\tau_{3}(t)\right) e^{-\int_{A_{3}(t)-\tau_{3}(t)}^{A_{3}(t)} \mu\left(t-A_{3}(t)+r, r\right) d r} \\
& =\rho\left(t-\tau_{3}(t), \tau_{2}\left(t-\tau_{3}(t)\right) e^{-\int_{t-\tau_{3}(t)}^{t} \mu_{3}\left(x_{3}(r)\right) d r}\right. \\
& =\rho\left(t-A_{3}(t), 0\right) e^{-\int_{0}^{\tau_{2}\left(t-\tau_{3}(t)\right)} \mu\left(t-A_{3}(t)+r, r\right) d r} e^{-\int_{t-\tau_{3}(t)}^{t} \mu_{3}\left(x_{3}(r)\right) d r} \\
& =\rho\left(t-A_{3}(t), 0\right) e^{-\int_{t-\tau_{3}(t)}^{t} \mu_{3}\left(x_{3}(r)\right) d r} e^{-\int_{t-A_{3}(t)}^{t-\tau_{3}(t)} \mu_{2}\left(x_{2}(r)\right) d r} \\
& =\rho\left(t-A_{3}(t), 0\right) e^{-\int_{t-\tau_{3}(t)}^{t} \mu_{3}\left(x_{3}(r)\right) d r} \alpha_{2}\left(t-\tau_{3}(t), t-A_{3}(t)\right) \\
& :=\rho\left(t-A_{3}(t), 0\right) \alpha_{3}\left(t, t-A_{3}(t)\right)
\end{aligned}
$$

where $\alpha_{2}\left(t, t-A_{2}(t)\right)=e^{-\int_{t-A_{2}(t)}^{t} \mu_{2}\left(x_{2}(r)\right) d r}$ and $\alpha_{3}\left(t, t-A_{3}(t)\right)=e^{-\int_{t-\tau_{3}(t)}^{t} \mu_{3}\left(x_{3}(r)\right) d r} \alpha_{2}\left(t-\tau_{3}(t), t-A_{3}(t)\right)$.

Similarly, we have

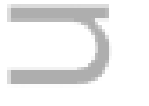

$$
\begin{aligned}
\rho\left(t, A_{i}(t)\right) & =\rho\left(t-A_{i}(t), 0\right) \alpha_{i}\left(t, t-A_{i}(t)\right) \\
& =\rho\left(t-A_{i}(t), 0\right) e^{-\int_{t-\tau_{i}(t)}^{t} \mu_{i}\left(x_{i}(r)\right) d r} \alpha_{i-1}\left(t-\tau_{i}(t), t-A_{i}(t)\right),
\end{aligned}
$$

where the iterative relationship of $\alpha_{i}\left(t, t-A_{i}(t)\right)$ is as follows $(i=2, \cdots, n)$

$$
\alpha_{i}\left(t, t-A_{i}(t)\right)=e^{-\int_{t-\tau_{i}(t)}^{t} \mu_{i}\left(x_{i}(r)\right) d r} \alpha_{i-1}\left(t-\tau_{i}(t), t-A_{i}(t)\right)
$$

with $\alpha_{1}=1$. Obviously, each $\alpha_{i}\left(t, t-A_{i}(t)\right)(i=2, \cdots, n)$ represents the density-dependent survival probability of an egg who was born at time $t-A_{i}(t)$ and is able to live until time $t$ when the egg eventually belongs to the stage $x_{i}$ with full maturation. In order for the model to be biologically meaningful, we prove the following lemma that concludes that the $i^{\text {th }}$ stage of the population will not go back to the previous $(i-1)^{\text {th }}$ stage except by birth. 
Lemma 1 With the assumption (H1), at time $t \geq 0$ for all $i=2, \cdots, n$, we have

(i) $A_{i}(t) \geq A_{i-1}(t)$ and $A_{i}(t)=\tau_{i}(t)+A_{i-1}\left(t-\tau_{i}(t)\right)$;

(ii) $1-A_{i}^{\prime}(t) \geq 0$ and $1-A_{i}^{\prime}(t)=\left(1-\tau_{i}^{\prime}(t)\right)\left(1-A_{i-1}^{\prime}\left(t-\tau_{i}(t)\right)\right)$.

Proof. Since $t-\tau_{3}(t)<t, t-\tau_{2}(t)$ is an non-decreasing function, we have $t-\tau_{3}(t)-\tau_{2}\left(t-\tau_{3}(t)\right) \leq t-\tau_{2}(t)$. This is equivalent to $A_{3}(t) \geq A_{2}(t)$. Again $t-\tau_{4}(t)<t, t-\tau_{3}(t)$ is a non-decreasing function, we have $t-\tau_{4}(t)-\tau_{3}\left(t-\tau_{4}(t)\right) \leq$ $t-\tau_{3}(t)$. Since $t-\tau_{2}(t)$ is non-decreasing, we have

$$
t-\tau_{4}(t)-\tau_{3}\left(t-\tau_{4}(t)\right)-\tau_{2}\left(t-\tau_{4}(t)-\tau_{3}\left(t-\tau_{4}(t)\right)\right) \leq t-\tau_{3}(t)-\tau_{2}\left(t-\tau_{3}(t)\right) .
$$

That is, $A_{4}(t) \geq A_{3}(t)$. By the same argument, we obtain $A_{i}(t) \geq A_{i-1}(t), \quad i=2, \cdots, n$. From equation (9), we can easily obtain $A_{i}(t)=\tau_{i}(t)+A_{i-1}\left(t-\tau_{i}(t)\right)$ and $1-A_{i}^{\prime}(t)=\left(1-\tau_{i}^{\prime}(t)\right)\left(1-A_{i-1}^{\prime}\left(t-\tau_{i}(t)\right)\right) \geq 0$

Then when $t>A_{n}(t)$, the closed form of the model becomes

$$
\begin{aligned}
x_{1}^{\prime}= & \delta \alpha_{n}\left(t, t-A_{n}(t)\right) b\left(x_{1}\left(t-A_{n}(t)\right)\right)\left(1-A_{n}^{\prime}(t)\right)-\mu_{1}\left(x_{1}(t)\right) x_{1}(t), \\
x_{2}^{\prime}= & b\left(x_{1}(t)\right)-\alpha_{2}\left(t, t-A_{2}(t)\right) b\left(x_{1}\left(t-A_{2}(t)\right)\right)\left(1-A_{2}^{\prime}(t)\right)-\mu_{2}\left(x_{2}(t)\right) x_{2}(t), \\
x_{i}^{\prime}= & \alpha_{i-1}\left(t, t-A_{i-1}(t)\right) b\left(x_{1}\left(t-A_{i-1}(t)\right)\right)\left(1-A_{i-1}^{\prime}(t)\right) \\
& -\alpha_{i}\left(t, t-A_{i}(t)\right) b\left(x_{1}\left(t-A_{i}(t)\right)\right)\left(1-A_{i}^{\prime}(t)\right)-\mu_{i}\left(x_{i}(t)\right) x_{i}(t), i=3, \cdots, n,
\end{aligned}
$$

where the birth function $b(x)$ and death function $\mu(x)$ are specified in assumptions $(\mathrm{H} 2)$ and $(\mathrm{H} 3), \alpha_{i}\left(t, t-A_{i}(t)\right)$ refers to Eq. (14), and relation $A_{i}(t)$ refers to lemma (1).

Note that each equation of $x_{i}^{\prime}(t)$ except $x_{1}^{\prime}(t)$ has the following form

$$
x_{i}^{\prime}(t)+\text { death rate }=\text { inflow rate }- \text { outflow rate }:=f_{\text {in }}(t)-f_{\text {out }}(t)
$$

"Inflow rate" indicates that at time $t$ all individuals enter the specific stage $\left(x_{i}\right)$ with zero stage-specific age at a rate $f_{\text {in }}(t)$; and "outflow rate" represents that all individuals leave the specific stage $\left(x_{i}\right)$ at full specific-stage maturity age at a rate $f_{\text {out }}(t)$. Moreover,

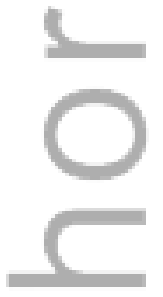

$$
\begin{aligned}
f_{\text {out }}(t)= & \alpha_{i}\left(t, t-A_{i}(t)\right) b\left(x_{1}\left(t-A_{i}(t)\right)\right)\left(1-A_{i}^{\prime}(t)\right) \\
= & e^{-\int_{t-\tau_{i}(t)}^{t} \mu_{i}\left(x_{i}(r)\right) d r} \alpha_{i-1}\left(t-\tau_{i}(t), t-\tau_{i}(t)-A_{i-1}\left(t-\tau_{i}(t)\right)\right) \\
& \cdot b\left(x_{1}\left(t-\tau_{i}(t)-A_{i-1}\left(t-\tau_{i}(t)\right)\right)\right)\left(1-\tau_{i}^{\prime}(t)\right)\left(1-A_{i-1}^{\prime}\left(t-\tau_{i}(t)\right)\right) \\
= & \left(1-\tau_{i}^{\prime}(t)\right) e^{-\int_{t-\tau_{i}(t)}^{t} \mu_{i}\left(x_{i}(r)\right) d r} f_{\text {in }}\left(t-\tau_{i}(t)\right) .
\end{aligned}
$$

Namely, $f_{\text {in }}(t)$ is related to $f_{\text {out }}(t)$ by the following form:

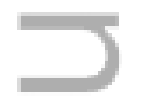

$$
f_{\text {out }}(t)=\left(1-\tau_{i}^{\prime}(t)\right) \sigma\left(t, t-\tau_{i}(t)\right) f_{\text {in }}\left(t-\tau_{i}(t)\right),
$$

where $\left(1-\tau_{i}^{\prime}(t)\right)$ is the "maturation ratio" of the population at the specific stage $x_{i}$ tracking entering and leaving of the stage, and $\sigma\left(t, t-\tau_{i}(t)\right):=e^{-\int_{t-\tau_{i}(t)}^{t} \mu_{i}\left(x_{i}(r)\right) d r}$ the survival probabilities from the moment entering the stage to the moment leaving the stage. We also emphasize that all inflow rates and outflow rates are time-dependent, and our model does not exclude the situation in which individuals may undergo no development in a low temperature condition or enter diapause induced by environmental condition such as photoperiod change provided that the "maturation ratio" $\left(1-\tau_{i}^{\prime}(t)\right)$ is zero.

\subsection{Nonnegativity and boundedness}

We consider solutions to system (15) with a focus on the long-term dynamics where $t \geq A_{n}(t)$. The initial data for system (15) cannot be arbitrary. For biological reasons the initial data must satisfy several constraints and we only consider solutions 
that satisfy these constraints. Define $\tau_{m}=\min _{t \in[0, \omega]} A_{n}(t), \tau_{M}=\max _{t \in[0, \omega]} A_{n}(t)$. It is easy to see that each exponential function $\alpha_{i}\left(t, t-A_{i}(t)\right)$ is always positive and $b\left(x_{1}(t)\right)$ is nonnegative provided that $x_{1}(t)$ is nonnegative.

Theorem 1 Let the initial data $x_{i}(\theta) \geq 0$ for $-\tau_{M} \leq \theta<0$ for each stage, and

$$
\begin{aligned}
x_{i}(0) & =\int_{-\tau_{i}(0)}^{0} e^{-\int_{s}^{0} \mu_{i}\left(x_{i}(r)\right) d r} \alpha_{i-1}\left(s, s-A_{i-1}(s)\right) b\left(x_{1}\left(s-A_{i-1}(s)\right)\right)\left(1-A_{i-1}^{\prime}(s)\right) d s \\
& =\int_{-\tau_{i}(0)}^{0} e^{-\int_{s}^{0} \mu_{i}\left(x_{i}(r)\right) d r} f_{i n}(s) d s, \quad i=2, \cdots, n
\end{aligned}
$$

Then each component $x_{i}(t)$ of the solution of the system (15) remains nonnegative for all $t \geq 0, i=1, \cdots, n$. Furthermore, each component of the solution is also bounded for all $t>0$.

Proof. First we show that $x_{1}(t) \geq 0$ for all $t \geq-\tau_{M}$ when $x_{1}(\theta) \geq 0$ for $-\tau_{M} \leq \theta \leq 0$. We prove Theorem 1 by showing that $x_{i}(t, \varepsilon)$ is the solution of the modified system obtained from system (15) by adding $\varepsilon$ to the right hand side with $\varepsilon$ being arbitrarily small. To show that $x_{1}(t, \varepsilon) \geq 0$ for all $t>0$, we suppose that $x_{1}(t, \varepsilon)<0$ for some $t>0$. Let $t^{*}=\inf \left\{t: t>0\right.$ and $x_{1}(t, \varepsilon)<$ $0\}$. Then $t^{*} \geq 0, x_{1}\left(t^{*}, \varepsilon\right)=0$ and $x_{1}^{\prime}\left(t^{*}, \varepsilon\right) \leq 0$. But, from the first equation of the modified system

$$
\begin{aligned}
x_{1}^{\prime}\left(t^{*}, \varepsilon\right)= & \delta \alpha_{n}\left(t^{*}, t^{*}-A_{n}\left(t^{*}\right)\right) b\left(x_{1}\left(t^{*}-A_{n}\left(t^{*}\right), \varepsilon\right)\right)\left(1-A_{n}^{\prime}\left(t^{*}\right)\right) \\
& -\mu_{1}\left(x_{1}\left(t^{*}, \varepsilon\right)\right) x_{1}\left(t^{*}, \varepsilon\right)+\varepsilon \\
= & \delta \alpha_{n}\left(t^{*}, t^{*}-A_{n}\left(t^{*}\right)\right) b\left(x_{1}\left(t^{*}-A_{n}\left(t^{*}\right), \varepsilon\right)\right)\left(1-A_{n}^{\prime}\left(t^{*}\right)\right)+\varepsilon
\end{aligned}
$$

Moreover, $A_{n}\left(t^{*}\right)>0$ ensures $t^{*}-A_{n}\left(t^{*}\right)<t^{*}$, implying that $x_{1}\left(t^{*}-A_{n}\left(t^{*}\right), \varepsilon\right) \geq 0$ by the definition of $t^{*}$. This, in turn, implies that $x_{1}^{\prime}\left(t^{*}, \varepsilon\right) \geq \varepsilon>0$, giving rise to a contradiction. Therefore, $x_{1}(t, \varepsilon) \geq 0$ for each $t>0$. This is true for arbitrarily small $\varepsilon>0$. Letting $\varepsilon \rightarrow 0$ gives $x_{1}(t) \geq 0$ as a solution of system (15).

Next we show the nonnegativity of $x_{i}(t)$ for all $t \geq 0, i=2, \cdots, n$. Moving the term $\mu_{i}\left(x_{i}(t)\right) x_{i}(t)$ to the left side of the equations of system (15) and multiplying both sides by $e^{\int_{0}^{t} \mu_{i}\left(x_{i}(r)\right) d r}$ yields

$$
\begin{aligned}
& \left(e^{\int_{0}^{t} \mu_{i}\left(x_{i}(r)\right) d r} x_{i}(t)\right)^{\prime} \\
= & e^{\int_{0}^{t} \mu_{i}\left(x_{i}(r)\right) d r}\left(f_{\text {in }}(t)-f_{\text {out }}(t)\right) \\
= & e^{\int_{0}^{t} \mu_{i}\left(x_{i}(r)\right) d r}\left(f_{\text {in }}(t)-\left(1-\tau_{i}^{\prime}(t)\right) e^{\int_{t-\tau_{i}(t)}^{t} \mu_{i}\left(x_{i}(r)\right) d r} f_{\text {in }}\left(t-\tau_{i}(t)\right)\right) \\
= & \left(\int_{t-\tau_{i}(t)}^{t} e^{-\int_{0}^{s} \mu_{i}\left(x_{i}(r)\right) d r} \alpha_{i-1}\left(s, s-A_{i-1}(s)\right) b\left(x_{1}\left(s-A_{i-1}(s)\right)\right)\left(1-A_{i-1}^{\prime}(s)\right) d s\right)^{\prime} .
\end{aligned}
$$

Combining the initial data constraints (18), equation (19) yields

$$
x_{i}(t)=\int_{t-\tau_{i}(t)}^{t} e^{-\int_{s}^{t} \mu_{i}\left(x_{i}(r)\right) d r} \alpha_{i-1}\left(s, s-A_{i-1}(s)\right) b\left(x_{1}\left(s-A_{i-1}(s)\right)\right)\left(1-A_{i-1}^{\prime}(s)\right) d s
$$

which is non-negative because of the nonnegativity of $x_{1}(t)$. The expression (20) is ecologically reasonable since it accounts for the total number of individuals in the specific stage $x_{i}$ at time $t$ who come from eggs laid at time $s-A_{i-1}(s)$ and successfully moult to the stage at time $s$ and still stay in the stage until time moment $t$. Here $b\left(x_{1}\left(s-A_{i-1}(s)\right)\right)$ represents the number of eggs who were born at time $s-A_{i-1}(s) ; \alpha_{i-1}\left(s, s-A_{i-1}(s)\right)$ is proportion of eggs who were born at time $s-A_{i-1}(s)$ and develop into $x_{i}$ stage at time moment $s ; e^{-\int_{s}^{t} \mu_{i}\left(x_{i}(r)\right) d r}$ is the survival probability at $x_{i}$ stage from time moment $s$ to time moment $t$; and $\left(1-A_{i-1}^{\prime}(s)\right)$ is the "maturation ratio" comparing to newborn eggs at time $s$ when eggs already develop into stage $x_{i}$ with zero stage-specific age. The variable $\tau_{i}(t)$ is the required maturation time during stage $x_{i}$ at time $t$. Eq. (20) indicates each component of the system (15) is nonnegative for all $t \geq 0$.

The boundedness of the solution of system (15) is easy to show. Denote by $N(t)=\sum_{i=2}^{n} x_{i}(t)+\frac{1}{\delta} x_{1}(t)$ the total number of individuals at all stages. It is easy to see that the birth function is bounded when the size of egg-laying females is nonnegative 
since

$$
b\left(x_{1}(t)\right) \leq \frac{p e^{-1}}{s_{T}}:=b \max .
$$

Putting all equations of system (15) together yields

where

$$
N^{\prime}(t)=b\left(x_{1}(t)\right)-\left(\sum_{i=2}^{n} \mu_{i}\left(x_{i}(t)\right) x_{i}(t)+\frac{1}{\delta} \mu_{1}\left(x_{1}(t)\right) x_{1}(t)\right) \leq b \max -\mu N(t),
$$

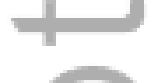

$$
\mu=\min _{j \in\{1, \cdots, n\}} \mu_{j}(0) .
$$

From (21) it follows that

$$
\text { (limsup } N(t) \leq b \max / \mu,
$$

which implies the boundedness of all solutions of system (15) subject to the initial condition constraints. This completes the proof.

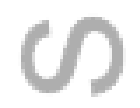

\section{Basic reproductive ratio $\left(\mathcal{R}_{0}\right)$}

\subsection{Definition of $\mathcal{R}_{0}$}

System (15) has a population-extinction (trivial) equilibrium. Linearizing system (15) at the population-extinction equilibrium yields

$$
\begin{aligned}
x_{1}^{\prime} & \left.=\delta \hat{\alpha}_{n}(t) p x_{1}\left(t-A_{n}(t)\right)\right)\left(1-A_{n}^{\prime}(t)\right)-\mu_{1}(0) x_{1}(t), \\
x_{2}^{\prime} & =p x_{1}(t)-\hat{\alpha}_{2}(t) p x_{1}\left(t-A_{2}(t)\right)\left(1-A_{2}^{\prime}(t)\right)-\mu_{2}(0) x_{2}(t), \\
x_{i}^{\prime} & =\hat{\alpha}_{i-1}(t) p x_{1}\left(t-A_{i-1}(t)\right)\left(1-A_{i-1}^{\prime}(t)\right)-\hat{\alpha}_{i}(t) p x_{1}\left(t-A_{i}(t)\right)\left(1-A_{i}^{\prime}(t)\right)-\mu_{i}(0) x_{i}(t)(i=3, \cdots, n),
\end{aligned}
$$

where $\hat{\alpha}_{i}(t)$ is the survival probability near the population-extinction equilibrium given by the following iteration relation

$$
\hat{\alpha}_{2}(t)=e^{-\mu_{2}(0) \tau_{2}(t)}, \quad \hat{\alpha}_{i}(t)=e^{-\mu_{i}(0) \tau_{i}(t)} \hat{\alpha}_{i-1}\left(t-\tau_{i}(t)\right), \quad i=3, \cdots, n
$$

Note that $\hat{\alpha}_{i}$ is a $\omega$-periodic function, i.e., $\hat{\alpha}_{i}(t+\omega)=\hat{\alpha}_{i}(t)$.

System (23) has a 1-dimensional decoupled subsystem

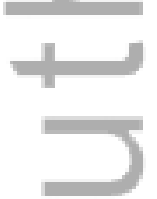

$$
\begin{aligned}
x_{1}^{\prime}(t) & =\delta p \hat{\alpha}_{n}(t)\left(1-A_{n}^{\prime}(t)\right) x_{1}\left(t-A_{n}(t)\right)-\mu_{1}(0) x_{1}(t) \\
& :=a(t) x_{1}\left(t-A_{n}(t)\right)-\mu_{1}(0) x_{1}(t)
\end{aligned}
$$

where

$$
a(t)=\delta p \hat{\alpha}_{n}(t)\left(1-A_{n}^{\prime}(t)\right)
$$

The rate of change of egg-laying females at time $t$ depends on the number of egg-laying females at time $t-A_{n}(t)$. To proceed further, we need the following assumption:

(H4) $h(t):=t-A_{n}(t)$ is a strictly increasing function of $t$.

We next identify $\mathcal{R}_{0}$. To this end, we examine the number of newly generated egg-laying females per unit time at time $t$. With the assumption (H4), at time $t$, the cohort of egg-laying female (with its size denoted by $x_{1}(t)$ ) will produce some newborns who will eventually become egg-laying females at the future time $h^{-1}(t):=\tilde{t}$, where $h(\tilde{t})=\tilde{t}-A_{n}(\tilde{t})$ is a strictly increasing 
function of $\tilde{t}$. We note that

$$
\begin{aligned}
\frac{d}{d t} x_{1}(\tilde{t}) & =\frac{d}{d \tilde{t}} x_{1}(\tilde{t}) \frac{d \tilde{t}}{d t}=\left[a(\tilde{t}) x_{1}(h(\tilde{t}))-\mu_{1}(0) x_{1}(\tilde{t})\right] \frac{1}{1-A_{n}^{\prime}(\tilde{t})} \\
& =\left[a\left(h^{-1}(t)\right) x_{1}(t)-\mu_{1}(0) x_{1}\left(h^{-1}(t)\right)\right] \frac{1}{1-A_{n}^{\prime}\left(h^{-1}(t)\right)} .
\end{aligned}
$$

That is, the number of newly generated egg-laying females per unit time at time $t$ is given by $y(t)=c(t) x_{1}(t)$ with $c(t):=a\left(h^{-1}(t)\right) /\left(1-A_{n}^{\prime}\left(h^{-1}(t)\right)\right)$. Note that

$$
\begin{aligned}
\left(e^{\mu_{1}(0) t} x_{1}(t)\right)^{\prime} & =e^{\mu_{1}(0) t}\left(\mu_{1}(0) x_{1}(t)+x_{1}^{\prime}(t)\right) \\
& =e^{\mu_{1}(0) t}\left(\mu_{1}(0) x_{1}(t)+a(t) x_{1}\left(t-A_{n}(t)\right)-\mu_{1}(0) x_{1}(t)\right) \\
& =e^{\mu_{1}(0) t} a(t) x_{1}\left(t-A_{n}(t)\right) .
\end{aligned}
$$

Integrating of (28) from $-\infty$ to $t$ yields

$$
x_{1}(t)=\int_{-\infty}^{t} e^{-\mu_{1}(0)(t-s)} a(s) x_{1}\left(s-A_{n}(s)\right) d s .
$$

Multiplying (29) by $c(t)$ gives (note $\left.c\left(s-A_{n}(s)\right)=\frac{a(s)}{1-A_{n}^{\prime}(s)}\right)$

where

$$
\begin{aligned}
y(t) & =c(t) \int_{-\infty}^{t} e^{-\mu_{1}(0)(t-s)} \frac{a(s)}{c\left(s-A_{n}(s)\right)} y\left(s-A_{n}(s)\right) d s \\
& =\int_{-\infty}^{t} c(t)\left(1-A_{n}^{\prime}(s)\right) e^{-\mu_{1}(0)(t-s)} y\left(s-A_{n}(s)\right) d s \\
& =\int_{A_{n}(t)}^{\infty} c(t) e^{-\mu_{1}(0)\left(t-h^{-1}(t-r)\right)} y(t-r) d r \\
& =\int_{0}^{\infty} \mathcal{K}(t, r) y(t-r) d r
\end{aligned}
$$

$$
\mathcal{K}(t, r)=\left\{\begin{array}{cc}
\delta p \hat{\alpha}_{n}\left(h^{-1}(t)\right) e^{-\mu_{1}(0)\left(t-h^{-1}(t-r)\right)} & , \quad r \geq A_{n}(t), \\
0 & , \quad r<A_{n}(t) .
\end{array}\right.
$$

In particular, $\mathcal{K}(t, r)$ is a periodic function with respect to time $t$, i.e., $\mathcal{K}(t, r)=\mathcal{K}(t+\omega, r)$. Moreover, the kernel $\mathcal{K}(t, r)$ has a biological interpretation. At time $t$, only the cohort of egg-laying females who are still alive before time $t-A_{n}(t)$ is capable of reproducing eggs which will mature to new generation of egg-laying females.

In Jagers and Nerman [17] and Thieme [34], the solution $y(t)$ of (30) of the form $e^{\lambda t} u(t)$ is considered, where $u(t)$ is a periodic function with period $\omega$ and satisfies

$$
u(t)=\int_{0}^{\infty} \mathcal{K}(t, r) e^{-\lambda r} u(t-r) d r .
$$

Define the $\omega$-periodic continuous eigenfunction space by $\mathcal{C}_{\omega}:=\{u: \mathbb{R} \rightarrow \mathbb{R}, u(t+\omega)=u(t)\}$ which is equipped with maximum norm $\|\cdot\|$, and define an integral operator $\mathcal{L}: \mathcal{C}_{\omega} \rightarrow \mathcal{C}_{\omega}$ by

$$
(\mathcal{L} u)(t)=\int_{0}^{\infty} \mathcal{K}(t, r) u(t-r) d r
$$

Lemma 2 The operator $\mathcal{L}$ is strongly positive, continuous and compact on $\mathcal{C}_{\omega}$.

Proof. It is clear that $\mathcal{L}$ is strongly positive in the sense that $(\mathcal{L} u)(t)>0$ if $u(t) \geq 0$ but $u(t) \neq 0$ for all $t \in \mathbb{R}$. According to the assumption ( $\mathbf{H} \mathbf{4})$, there exist a positive number $\theta>0$ such that

$$
h^{-1}\left(t-A_{n}(t)\right)-h^{-1}(t-r) \geq \theta\left(t-A_{n}(t)-t+r\right)=\theta\left(r-A_{n}(t)\right)
$$


In terms of (24) and (34), we have

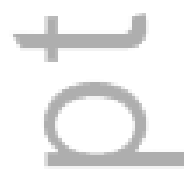

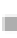

$$
\begin{aligned}
\|(\mathcal{L} u)(t)\| & =\left\|\int_{A_{n}(t)}^{\infty} \delta p \hat{\alpha}_{n}\left(h^{-1}(t)\right) e^{-\mu_{1}(0)\left(t-h^{-1}(t-r)\right)} u(t-r) d r\right\| \\
& =\left\|\int_{A_{n}(t)}^{\infty} \delta p \hat{\alpha}_{n}\left(h^{-1}(t)\right) e^{-\mu_{1}(0)\left(h^{-1}\left(t-A_{n}(t)\right)-h^{-1}(t-r)\right)} u(t-r) d r\right\| \\
& \leq\left\|\int_{A_{n}(t)}^{\infty} \delta p e^{-\mu_{1}(0) \theta\left(r-A_{n}(t)\right)} d r\right\|\|u\| \\
& =\delta p e^{-\mu_{1}(0) \theta A_{n}(t)} \frac{1}{\mu_{1}(0) \theta} e^{-\mu_{1}(0) \theta A_{n}(t)}\|u\| \\
& =\frac{\delta p}{\mu_{1}(0) \theta}\|u\| .
\end{aligned}
$$

It is easy to see from (35) that operator $\mathcal{L}$ is bounded, and thereby, continuous on $\mathcal{C}_{\omega}$. Since

$$
\begin{aligned}
(\mathcal{L} u)(t) & =\int_{A_{n}(t)}^{\infty} \delta p \hat{\alpha}_{n}\left(h^{-1}(t)\right) e^{-\mu_{1}(0)\left(t-h^{-1}(t-r)\right)} u(t-r) d r \\
& =\int_{-\infty}^{t} \delta p \hat{\alpha}_{n}\left(h^{-1}(t)\right) e^{-\mu_{1}(0)\left(t-h^{-1}\left(s-A_{n}(s)\right)\right)} u\left(s-A_{n}(s)\right)\left(1-A_{n}^{\prime}(s)\right) d s
\end{aligned}
$$

and

$$
\frac{d}{d t} \hat{\alpha}_{n}(t)=\hat{\alpha}_{n}(t) g_{1}(t), \quad \frac{d}{d t} h^{-1}(t)=\frac{1}{1-A_{n}^{\prime}\left(h^{-1}(t)\right)}:=g_{2}(t)>0
$$

for some $\omega$-periodic functions $g_{1}(t)$ and $g_{2}(t)$ because $\hat{\alpha}_{n}(t)$ is an exponential $\omega$-periodic function (refer to (24)). Then we have

$$
\begin{aligned}
\frac{d}{d t}(\mathcal{L} u)(t) & =\delta p \hat{\alpha}_{n}\left(h^{-1}(t)\right)\left(1-A_{n}^{\prime}(t)\right) u\left(t-A_{n}(t)\right)+\left(g_{1}\left(h^{-1}(t)\right) g_{2}(t)-\mu_{1}(0)\right)(\mathcal{L} u)(t) \\
& :=F(t) u\left(t-A_{n}(t)\right)+G(t)(\mathcal{L} u)(t),
\end{aligned}
$$

where $F(t)=\delta p \hat{\alpha}_{n}\left(h^{-1}(t)\right)\left(1-A_{n}^{\prime}(t)\right)>0$ is positive and periodic, and $G(t)=g_{1}\left(h^{-1}(t)\right) g_{2}(t)-\mu_{1}(0)$ is periodic. It the follows that for any $b>0$ such that $\|u\| \leq b$, there exists a positive $B=B(b)>0$ such that $\left|\frac{d}{d t}(\mathcal{L} u)(t)\right| \leq B$ for all $t \in[0, \omega]$ and $u \in \mathcal{C}_{\omega}$ with $\|u\| \leq b$. Thus, the Ascoli-Arzela theorem implies that $\mathcal{L}$ is compact on $\mathcal{C}_{\omega}$.

On page 77 of [11], $\mathcal{L}$ is called the "next generation operator". Following Bacaër [3], Bacaër and Guernaoui [2], and Wang and Zhao [35], we define the basic reproductive ratio as the spectral radius of the linear integral operator acting on the same function space of $\omega$-periodic continuous functions, i.e.,

$$
\mathcal{R}_{0}=\rho(\mathcal{L})
$$

Theorem 2 When $\mathcal{R}_{0}<1$, then zero solution of system (15) is locally asymptotically stable; when $\mathcal{R}_{0}>1$, zero solution of system (15) is unstable.

Proof. By Lemma 2 and the Krein-Rutman theorem of strongly positive and compact linear operator, we can conclude that the spectral radius of $\mathcal{L}$ is a simple positive eigenvalue of $\mathcal{L}$ with an positive eigenvector in $\mathcal{C}_{\omega}$, and all other eigenvalue in absolute value is strictly less than the spectral radius of $\mathcal{L}$, so that $\mathcal{R}_{0}>0$. As shown the statement in the page 427 of [2], we obtain $\lambda>0$ if $\mathcal{R}_{0}>1$ and $\lambda<0$ if $\mathcal{R}_{0}<1$, which means zero solution is local asymptotically stable if $\mathcal{R}_{0}<1$ and it is unstable if $\mathcal{R}_{0}>1$.

\subsection{Calculation of $\mathcal{R}_{0}$}

There are algorithms developed to calculate $\mathcal{R}_{0}$ numerically [3]. Here we propose an algorithm by using the most intuitive discretization and integration. This will link the calculation of $\mathcal{R}_{0}$ to the calculation of the spectral radius of a Leslie matrix. 
Changing the variable $\theta=t-r$ of (33), we obtain

$$
\begin{aligned}
(\mathcal{L} u)(t) & =\int_{0}^{\infty} \mathcal{K}(t, r) u(t-r) d r \\
& =\delta p \hat{\alpha}_{n}\left(h^{-1}(t)\right) e^{-\mu_{1}(0) t} \int_{A_{n}(t)}^{\infty} e^{\mu_{1}(0) h^{-1}(t-r)} u(t-r) d r \\
& :=\bar{p}(t) \int_{-\infty}^{t-A_{n}(t)} e^{\mu_{1}(0) h^{-1}(\theta)} u(\theta) d \theta \\
& =\bar{p}(t)\left[\int_{0}^{t-A_{n}(t)} e^{\mu_{1}(0) h^{-1}(\theta)} u(\theta) d \theta+\int_{-\infty}^{0} e^{\mu_{1}(0) h^{-1}(\theta)} u(\theta) d \theta\right]
\end{aligned}
$$

where

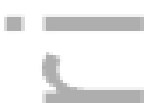

$$
\bar{p}(t)=\delta p \hat{\alpha}_{n}\left(h^{-1}(t)\right) e^{-\mu_{1}(0) t} .
$$

Since $u(t)$ is $\omega$-periodic, we have

$$
\begin{aligned}
\int_{-\infty}^{0} e^{\mu_{1}(0) h^{-1}(\theta)} u(\theta) d \theta & =\sum_{m=0}^{\infty} \int_{-(m+1) \omega}^{-m \omega} e^{\mu_{1}(0) h^{-1}(\theta)} u(\theta) d \theta \\
& =\int_{0}^{\omega} \sum_{m=0}^{\infty} e^{\mu_{1}(0) h^{-1}(\theta-(m+1) \omega} u(\theta) d \theta .
\end{aligned}
$$

So equation (37) is equivalent to

$$
\begin{aligned}
(\mathcal{L} u)(t)= & \bar{p}(t)\left[\int_{0}^{t-A_{n}(t)} e^{\mu_{1}(0) h^{-1}(\theta)} u(\theta) d \theta+\int_{0}^{\omega} \sum_{m=0}^{\infty} e^{\mu_{1}(0) h^{-1}(\theta-(m+1) \omega)} u(\theta) d \theta\right] \\
= & \bar{p}(t)\left[\int_{0}^{t-A_{n}(t)} e^{\mu_{1}(0) h^{-1}(\theta)} u(\theta) d \theta+\int_{0}^{t-A_{n}(t)} \sum_{m=0}^{\infty} e^{\mu_{1}(0) h^{-1}(\theta-(m+1) \omega)} u(\theta) d \theta\right. \\
& \left.+\int_{t-A_{n}(t)}^{\omega} \sum_{m=0}^{\infty} e^{\mu_{1}(0) h^{-1}(\theta-(m+1) \omega)} u(\theta) d \theta\right] \\
= & \bar{p}(t)\left[\int_{0}^{t-A_{n}(t)} \sum_{m=0}^{\infty} e^{\mu_{1}(0) h^{-1}(\theta-m \omega)} u(\theta) d \theta+\int_{t-A_{n}(t)}^{\omega} \sum_{m=0}^{\infty} e^{\mu_{1}(0) h^{-1}(\theta-\omega-m \omega)} u(\theta) d \theta\right] \\
:= & \bar{p}(t)\left[\int_{0}^{t-A_{n}(t)} H(\theta) u(\theta) d \theta+\int_{t-A_{n}(t)}^{\omega} H(\theta-\omega) u(\theta) d \theta\right]
\end{aligned}
$$

with

$$
H(\theta)=\sum_{m=0}^{\infty} e^{\mu_{1}(0) h^{-1}(\theta-m \omega)} .
$$

In Equation (38), the integral is over an interval of one period $[0, \omega]$ and $u(t)$ is an $\omega$-periodic function. To compute $\mathcal{R}_{0}$ numerically, we partition the interval $[0, \omega]$ into $N$ (a large integer) subintervals of equal length. Set $t_{i}=(i-1) \omega / N$ for $i=1,2, \cdots, N$. Then at the point $t_{i}$, equation (38) becomes

$$
(\mathcal{L} u)\left(t_{i}\right)=\bar{p}\left(t_{i}\right)\left[\int_{0}^{t_{i}-A_{n}\left(t_{i}\right)} H(\theta) u(\theta) d \theta+\int_{t_{i}-A_{n}\left(t_{i}\right)}^{\omega} H(\theta-\omega) u(\theta) d \theta\right] .
$$

For each $t_{i} \in[0, \omega)$, there is a unique integer $k_{i}$ such that $t_{i}+k_{i} \omega-A_{n}\left(t_{i}\right) \in[0, \omega)$. Denote $l_{i}:=\left[\frac{t_{i}+k_{i} \omega-A_{n}\left(t_{i}\right)}{\frac{\omega}{N}}+1\right] \in$ $\{1,2, \cdots, N\}$, i.e., the nearest integer less than or equal to $\frac{t_{i}+k_{i} \omega-A_{n}\left(t_{i}\right)}{\frac{\omega}{N}}+1$. Replacing $t_{i}+k_{i} \omega$ by $t_{i}$ in equation (39), we 
obtain

$$
\begin{aligned}
(\mathcal{L} u)\left(t_{i}\right)= & \bar{p}\left(t_{i}+k_{i} \omega\right)\left[\int_{0}^{t_{i}+k_{i} \omega-A_{n}\left(t_{i}\right)} H(\theta) u(\theta) d \theta+\int_{t_{i}+k_{i} \omega-A_{n}\left(t_{i}\right)}^{\omega} H(\theta-\omega) u(\theta) d \theta\right] \\
= & \bar{p}\left(t_{i}+k_{i} \omega\right)\left[\int_{0}^{t_{l_{i}}} H(\theta) u(\theta) d \theta+\int_{t_{l_{i}}}^{t_{i}+k_{i} \omega-A_{n}\left(t_{i}\right)} H(\theta) u(\theta) d \theta\right. \\
& \left.+\int_{t_{i}+k_{i} \omega-A_{n}\left(t_{i}\right)}^{t_{i_{i}+1}} H(\theta-\omega) u(\theta) d \theta+\int_{t_{l_{i}+1}}^{\omega} H(\theta-\omega) u(\theta) d \theta\right] .
\end{aligned}
$$

In the case where $t_{i_{i}}=t_{i}+k_{i} \omega-A_{n}\left(t_{i}\right)$, equation (40) becomes

$$
\begin{aligned}
(\mathcal{L} u)\left(t_{i}\right) & =\bar{p}\left(t_{i}+k_{i} \omega\right)\left[\sum_{j=2}^{l_{i}} H\left(t_{j}\right) u\left(t_{j}\right) \frac{\omega}{N}+\sum_{j=l_{i}+1}^{N+1} H\left(t_{j}-\omega\right) u\left(t_{j}\right) \frac{\omega}{N}\right] \\
& =\bar{p}\left(t_{i}+k_{i} \omega\right)\left[\sum_{j=2}^{l_{i}} H\left(t_{j}\right) u\left(t_{j}\right) \frac{\omega}{N}+\sum_{j=l_{i}+1}^{N} H\left(t_{j}-\omega\right) u\left(t_{j}\right) \frac{\omega}{N}+H\left(t_{N+1}-\omega\right) u\left(t_{N+1}\right) \frac{\omega}{N}\right] \\
& =\bar{p}\left(t_{i}+k_{i} \omega\right)\left[\sum_{j=2}^{l_{i}} H\left(t_{j}\right) u\left(t_{j}\right) \frac{\omega}{N}+\sum_{j=l_{i}+1}^{N} H\left(t_{j}-\omega\right) u\left(t_{j}\right) \frac{\omega}{N}+H\left(t_{1}\right) u\left(t_{1} \frac{\omega}{N}\right)\right] \\
& =\bar{p}\left(t_{i}+k_{i} \omega\right)\left[\sum_{j=1}^{l_{i}} H\left(t_{j}\right) u\left(t_{j}\right) \frac{\omega}{N}+\sum_{j=l_{i}+1}^{N} H\left(t_{j}-\omega\right) u\left(t_{j}\right) \frac{\omega}{N}\right]
\end{aligned}
$$

In the case where $t_{l_{i}}<t_{i}+k_{i} \omega-A_{n}\left(t_{i}\right)$, equation (40) becomes

$$
\begin{aligned}
(\mathcal{L} u)\left(t_{i}\right)= & \bar{p}\left(t_{i}+k_{i} \omega\right)\left[\sum_{j=1}^{l_{i}-1} H\left(t_{j}\right) u\left(t_{j}\right) \frac{\omega}{N}+\int_{t_{l_{i}}}^{t_{i}+k_{i} \omega-A_{n}\left(t_{i}\right)} H(\theta) u(\theta) d \theta\right. \\
& \left.+\int_{t_{i}+k_{i} \omega-A_{n}\left(t_{i}\right)}^{t_{l_{i}+1}} H(\theta-\omega) u(\theta) d \theta+\sum_{j=l_{i}+1}^{N} H\left(t_{j}-\omega\right) u\left(t_{j}\right) \frac{\omega}{N}\right] \\
= & \bar{p}\left(t_{i}+k_{i} \omega\right)\left[\sum_{j=1}^{l_{i}-1} H\left(t_{j}\right) u\left(t_{j}\right) \frac{\omega}{N}+H\left(t_{l_{i}}\right) u\left(t_{l_{i}}\right) \frac{\omega}{N}+\sum_{j=l_{i}+1}^{N} H\left(t_{j}-\omega\right) u\left(t_{j}\right) \frac{\omega}{N}\right] \\
= & \bar{p}\left(t_{i}+k_{i} \omega\right)\left[\sum_{j=1}^{l_{i}} H\left(t_{j}\right) u\left(t_{j}\right) \frac{\omega}{N}+\sum_{j=l_{i}+1}^{N} H\left(t_{j}-\omega\right) u\left(t_{j}\right) \frac{\omega}{N}\right]
\end{aligned}
$$

Let $W_{i}=u\left(t_{i}\right)$. Then the problem of estimating $\mathcal{R}_{0}$ in (36) reduces to the calculation of the spectral radius of a given Leslie matrix. Namely, we have the matrix eigenvalue problem of the form $\tilde{\mathcal{R}}_{0} \mathbf{W}=\mathbf{x} \mathbf{W}$, where $\mathbf{W}=\left(W_{1}, W_{2}, \cdots, W_{N}\right)^{T}$, and $\tilde{\mathcal{R}}_{0}$ is the spectral radius of a $N \times N$ positive matrix $\mathbf{X}$. In this matrix, the $(i, j)$ element is given by

$$
x_{i j}=\left\{\begin{array}{l}
\delta p \hat{\alpha}_{n}\left(h^{-1}\left(t_{i}\right)\right) \frac{\omega}{N} \sum_{m=0}^{\infty} e^{-\mu_{1}(0)\left(t_{i}-h^{-1}\left(t_{j}-k_{i} \omega-m \omega\right)\right)}, \quad 1 \leq j \leq l_{i}, \\
\delta p \hat{\alpha}_{n}\left(h^{-1}\left(t_{i}\right)\right) \frac{\omega}{N} \sum_{m=0}^{\infty} e^{-\mu_{1}(0)\left(t_{i}-h^{-1}\left(t_{j}-k_{i} \omega-(m+1) \omega\right)\right)}, \quad l_{i}+1 \leq j \leq N .
\end{array}\right.
$$

Remark 1 Since $h(t)=t-A_{n}(t)$ is assumed to be a strictly increasing function with respect to $t$, we have the existence of $h^{-1}$, and it can be easily verified that $h^{-1}(t+m \omega)=h^{-1}(t)+m \omega, m \in \mathcal{Z}$. 


\subsection{Leslie Matrix $(\mathbf{X})$ in a periodic time delay environment}

In the formula (41), it is useful to rewrite $\mathbf{X}$ in the following form:

$$
\mathbf{X}=\left(\begin{array}{ccccccc}
r_{1} s_{1,1} & r_{1} s_{1,2} & \cdots & r_{1} s_{1, l_{1}} & r_{1} s_{1, l_{1}+1} & \cdots & r_{1} s_{1, N} \\
r_{2} s_{2,1} & r_{2} s_{2,2} & \cdots & r_{2} s_{2, l_{2}} & r_{2} s_{2, l_{2}+1} & \cdots & r_{2} s_{2, N} \\
\vdots & \vdots & & \vdots & \vdots & & \vdots \\
r_{N} S_{N, 1} & r_{N} S_{N, 2} & \cdots & r_{N} S_{N, l_{N}} & r_{N} S_{N, I_{N}+1} & \cdots & r_{N} S_{N, N}
\end{array}\right)
$$

The above matrix $\mathbf{X}$ has the following biological interpretations:

$=$

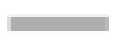

\section{(1)}

(a) $r_{i}=\delta p \hat{\alpha}_{n}\left(h^{-1}\left(t_{i}\right)\right) \frac{\omega}{N}(i=1, \cdots, N)$ is the number of newly generated egg-laying females per $\frac{\omega}{N}$-unit time at future time $h^{-1}\left(t_{i}\right)$ produced by an egg-laying female at time $t_{i}$;

(b) $l_{i}=\left[\frac{t_{i}+k_{i} \omega-A_{n}\left(t_{i}\right)}{\frac{\omega}{N}}+1\right] \in\{1,2, \cdots, N\}(i=1, \cdots, N)$, the nearest integer less than or equal to $\frac{t_{i}+k_{i} \omega-A_{n}\left(t_{i}\right)}{\frac{\omega}{N}}+1, w h e r e k_{i}$ is the unique integer such that $t_{i}+k_{i} \omega-A_{n}\left(t_{i}\right) \in[0, \omega)$;

(c) $s_{i, j}$ which is given below

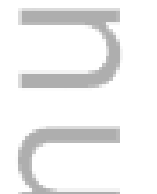

$$
s_{i, j}=\left\{\begin{array}{l}
e^{-\mu_{1}(0)\left(t_{i}-h^{-1}\left(t_{j}-k_{i} \omega\right)\right)} \frac{1}{1-e^{-\mu_{1}(0) \omega}}, \quad 1 \leq j \leq l_{i}, \\
e^{-\mu_{1}(0)\left(t_{i}-h^{-1}\left(t_{j}-k_{i} \omega-\omega\right)\right)} \frac{1}{1-e^{-\mu_{1}(0) \omega}}, \quad l_{i}+1 \leq j \leq N
\end{array}\right.
$$

represents the accumulated survival probability of all egg-laying females at time $t_{i}$ who developed from eggs at time $t_{j}-k_{i} \omega-m \omega$ or $t_{j}-k_{i} \omega-\omega-m \omega$, and will become egg-laying females at the future time $h^{-1}\left(t_{j}-k_{i} \omega-m \omega\right)$ or $h^{-1}\left(t_{j}-k_{i} \omega-\omega-m \omega\right)$, and have survived until the time $t_{i}$. This can be observed easily since $t_{i} \leq t_{i}+k_{i} \omega-A_{n}\left(t_{i}\right)$ and $t_{i_{i}}=t_{j}+\left(l_{i}-j\right) \omega / N$ implies

$$
\begin{aligned}
t_{j}-k_{i} \omega & =t_{l_{i}}-\left(I_{i}-j\right) \frac{\omega}{N}-k_{i} \omega \leq t_{i}+k_{i} \omega-A_{n}\left(t_{i}\right)-k_{i} \omega-\left(I_{i}-j\right) \frac{\omega}{N} \\
& =t_{i}-A_{n}\left(t_{i}\right)-\left(l_{i}-j\right) \frac{\omega}{N} \leq t_{i}-A_{n}\left(t_{i}\right), \quad j=1, \cdots, l_{i} ;
\end{aligned}
$$

and

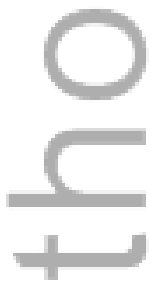

$$
\begin{aligned}
t_{j}-k_{i} \omega-\omega & =t_{l_{i}}-\left(I_{i}-j\right) \frac{\omega}{N}-k_{i} \omega-\omega \\
& \leq t_{i}+k_{i} \omega-A_{n}\left(t_{i}\right)-k_{i} \omega-\left(l_{i}-j\right) \frac{\omega}{N}-\omega \\
& =t_{i}-A_{n}\left(t_{i}\right)-\left(I_{i}-j+N\right) \frac{\omega}{N} \\
& <t_{i}-A_{n}\left(t_{i}\right), \quad j=l_{i}+1, \cdots, N .
\end{aligned}
$$

(d) $x_{i j}=r_{i} s_{i, j}\left(i=1,2, \cdots, N ; j=1, \cdots, l_{i}, l_{i}+1, \cdots, N\right)$ is the number of newly generated egg-laying females per $\frac{\omega}{N}$-unit time at time group $i$ caused from all previous generation individuals of egg-laying females of time group $j$.

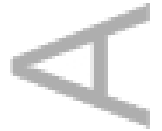

Comparing with the classical Leslie matrix, the females rate population was divided into $N$ groups in terms of the rat's age and each cell $(i, j)^{\text {th }}$ of the Leslie matrix accounts for how many individuals of female rats will be in the age group $i$ at the next time step from each individuals of group $j[7]$. Here, in a periodic environment, we divide the population of egg-laying females into $N$ groups in terms of time in a period $[0, \omega]$, thereby each cell $(i, j)^{\text {th }}$ of our matrix $\mathbf{X}$ indicates how many egg-laying females will be in the group $i$ at next generation step from all individuals of group $j$ at previous generation. Therefore, our $\mathbf{X}$ is just a result of applying the classical demographic Leslie matrix in a constant environment to the periodic environment. A crucial difference here is that the previous generation of egg-laying females is time-dependent. 
Table 1. Estimate $\mathcal{R}_{0} . N$ is number of points equally discretizing the interval $[0, \omega)$, which represents 1 year.

\begin{tabular}{ccccccc}
\hline$N$ & 100 & $365 \times 2$ & $365 \times 4$ & $365 \times 6$ & $365 \times 8$ & $365 \times 10$ \\
\hline $\mathcal{R}_{0}$ & 6.834 & 6.742 & 6.746 & 6.747 & 6.747 & 6.747 \\
\hline
\end{tabular}

\section{Numerical simulations}

In this section, we present some numerical simulation results using our proposed numerical algorithm for $\mathcal{R}_{0}$. We also use these results to examine the impact of the amplitude and the initial phase of the periodic delay, as well as the implications of temperature variation on the basic reproductive ratio $\mathcal{R}_{0}$.

In what follows, we let $\left[\mathcal{R}_{0}\right]$ be the basic reproductive ratio of the corresponding time-averaged autonomous delay differential system of (25). Such a system is obtained by replacing the periodic delay $\tau_{i}(t)$ with its corresponding time-averaged delay $\bar{\tau}_{i}=\frac{1}{\omega} \int_{0}^{\omega} \tau_{i}(t) d t=\tau_{i 0}$ for the original system of (25). This allows us to compare the basic reproductive ratio $\mathcal{R}_{0}$ in the periodic environment with $\left[\mathcal{R}_{0}\right]$ in the time-averaged constant environment. We also compare the value of $\mathcal{R}_{0}$ and $\left[\mathcal{R}_{0}\right]$ with the corresponding dominant Floquet multiplier and apply our developed method to calculate basic reproductive ratio of Ixodes scapularis tick population under variable temperature conditions.

\subsection{The impact of amplitude and phase of periodic delay on $\mathcal{R}_{0}$}

In this subsection, we demonstrate our numerical algorithm by considering two examples, one with two stages and another with four stages. The results can be conveniently used to examining the effect of the amplitude and the initial phase of the periodic delay on $\mathcal{R}_{0}$.

Example 1. We consider Nicholson blowflies equation which was proposed in [15] by Gurney et al. In the model, larvae become adults $\tau$ days after birth. The whole model system reads

$$
\left\{\begin{array}{l}
u_{i}^{\prime}(t)=p u_{m}(t) e^{-a u_{m}(t)}-e^{-\gamma \tau(t)}\left(1-\tau^{\prime}(t)\right) p u_{m}(t-\tau(t)) e^{-a u_{m}(t-\tau(t))}-\gamma u_{i}(t), \\
u_{m}^{\prime}(t)=\delta e^{-\gamma \tau(t)}\left(1-\tau^{\prime}(t)\right) p u_{m}(t-\tau(t)) e^{-a u_{m}(t-\tau(t))}-d u_{m}(t),
\end{array}\right.
$$

where $u_{i}$ is the number of immature blowflies, $u_{m}$ is the number of matured females blowflies, $p$ is the number of eggs laid per capita adult females blowflies, a measures the strength of density-dependence for fecundity, $\delta$ is the sex ratio, $\gamma$ and $d$ are mortality rates for immature and matured blowflies respectively. We assume $\tau$ is a periodic function given by

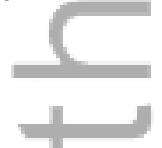

$$
\tau(t)=\tau_{0}\left(1+\epsilon \cos \left(\frac{2 \pi}{365}(t+\phi)\right)\right)
$$

Corresponding to the first equation in (23) is the linearization of the second equation in (43) at trivial solution, that is,

$$
u_{m}^{\prime}(t)=\delta e^{-\gamma \tau(t)}\left(1-\tau^{\prime}(t)\right) p u_{m}(t-\tau(t))-d u_{m}(t)
$$

which will be used to define and calculate $\mathcal{R}_{0}$ as shown in Section 3.

We first discuss the convergence of our proposed numerical method. We fix $\tau_{0}=15, \gamma=0.1, d=0.133, p=8, \delta=0.5$ similar to those in [26], and set $\epsilon=0.1, \phi=0, \omega=365$. We show in Table 1 the approximation of $\mathcal{R}_{0}$ which indicates the fast convergence of our proposed algorithm. We now conduct some numerical simulations to gain insights on the relationship between our basic reproductive ratio and the amplitude and phase of the periodic delay. Figure 1 shows that increasing the amplitude of the periodic delay increases the basic reproductive ratio $\mathcal{R}_{0}$, and that using the corresponding time-averaged delay differential system tends to underestimate the ratio $\left(\left[\mathcal{R}_{0}\right]<\mathcal{R}_{0}\right)$. Figure 2 shows that the basic reproduction ratio $\mathcal{R}_{0}$ remains at a constant value with varying $\phi$. Therefore, $\mathcal{R}_{0}$ is independent of the phase difference in the two stage-structured scenario.

Example 2. We consider Aedes Aegypti mosquito population consisting of four stages: egg, larva, pupa and adult females. We 


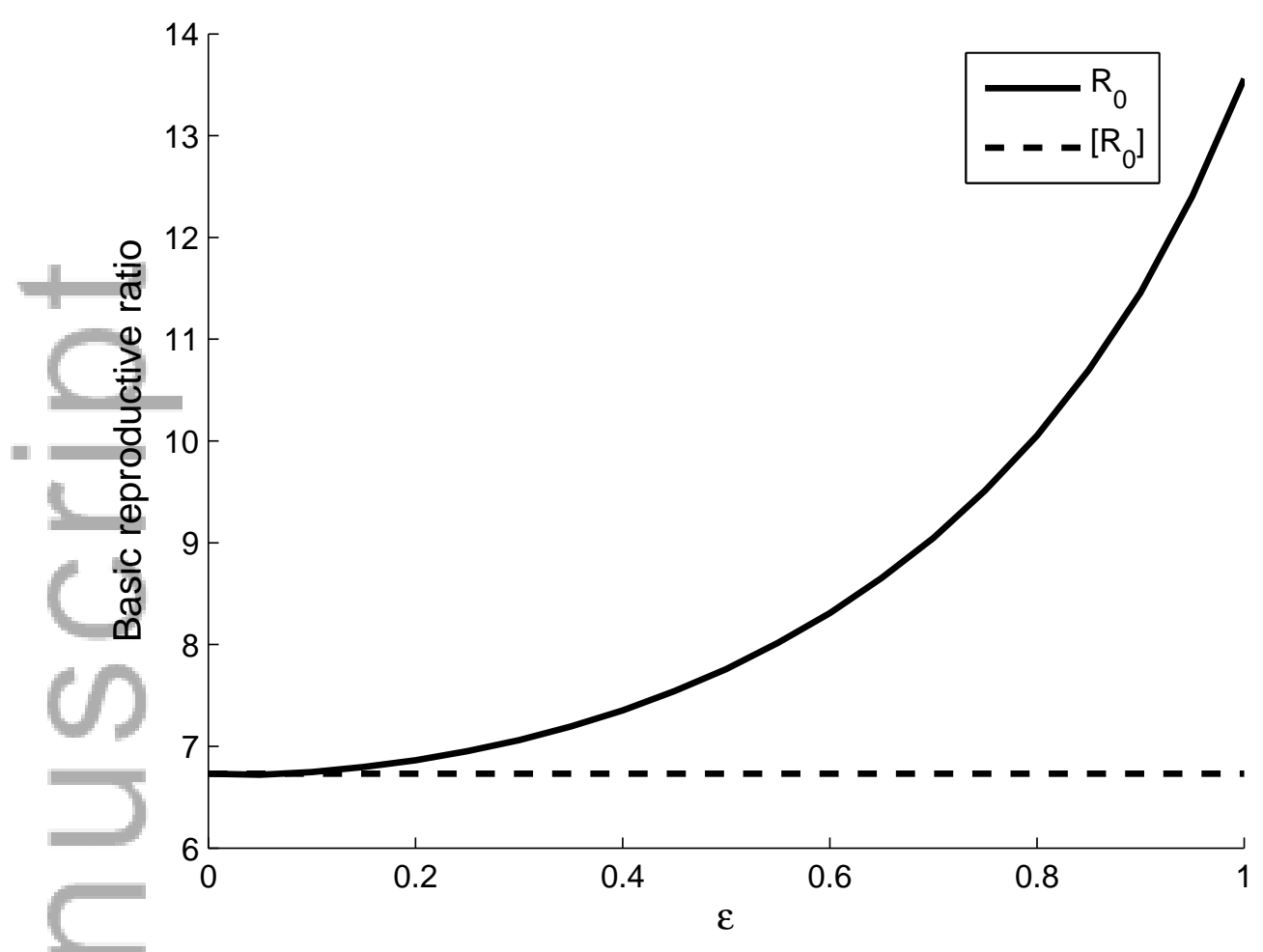

Figure 1. The graph of $\mathcal{R}_{0}$ versus $\epsilon$ with $\epsilon$ in $[0,1]$. Solid lines indicates the $\mathcal{R}_{0}$ values and dashed line is the corresponding time-averaged [ $\left.\mathcal{R}_{0}\right]$. Baseline parameter values: $\tau_{0}=15, \phi=0, \gamma=0.1, d=0.133, p=8, \delta=0.5, \omega=365$.

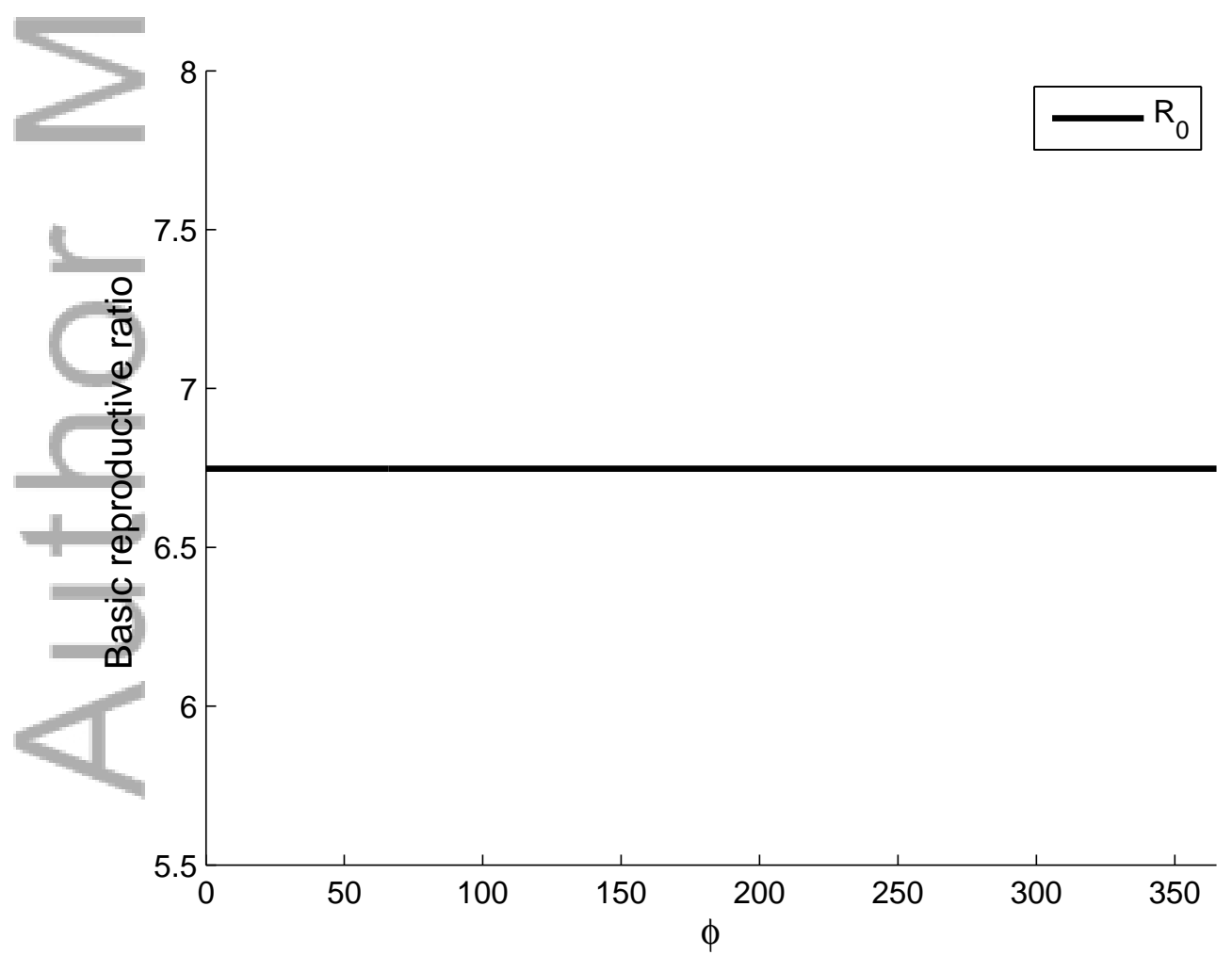

Figure 2. The graph of $\mathcal{R}_{0}$ versus $\phi$ with $\phi$ in [0,365]. Baseline parameter values: $\tau_{0}=15, \epsilon=0.1, \gamma=0.1, d=0.133, p=8, \delta=0.5, \omega=365$. 
set $\delta(=0.5)$ as the sex ratio and $p(=200 / 365)$ the average number of eggs laid per capita adult females per day. Mortalities of eggs, larvae, pupae and adults are set as $\mu_{2}=0.01, \mu_{3}=0.025, \mu_{4}=0.025$ and $\mu_{1}=0.09$ respectively taking from literature [27]. Average time delays from egg-to-larva, larva-to-pupa, pupa-to-adult are set as $\tau_{20}=5, \tau_{30}=10, \tau_{40}=2$ from website: http://www.denguevirusnet.com/life-cycle-of-aedes-aegypti.html. As Aedes Aegypti is affected by the temperature [27], we assume relevant time delays $\tau_{2}(t), \tau_{3}(t)$ and $\tau_{4}(t)$ take the forms

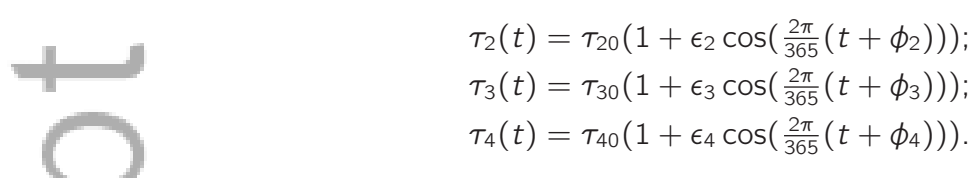

Linearizing system at mosquito-extinction equilibrium derived from first principle [37] yields

$$
x_{1}^{\prime}(t)=\delta p \alpha(t) x_{1}\left(t-A_{4}(t)\right)\left(1-A_{4}^{\prime}(t)\right)-\mu_{1} x_{1}(t)
$$

where $x_{1}$ is the number of adult females mosquitoes. $\alpha(t)$ and $A_{4}(t)$ have the following forms

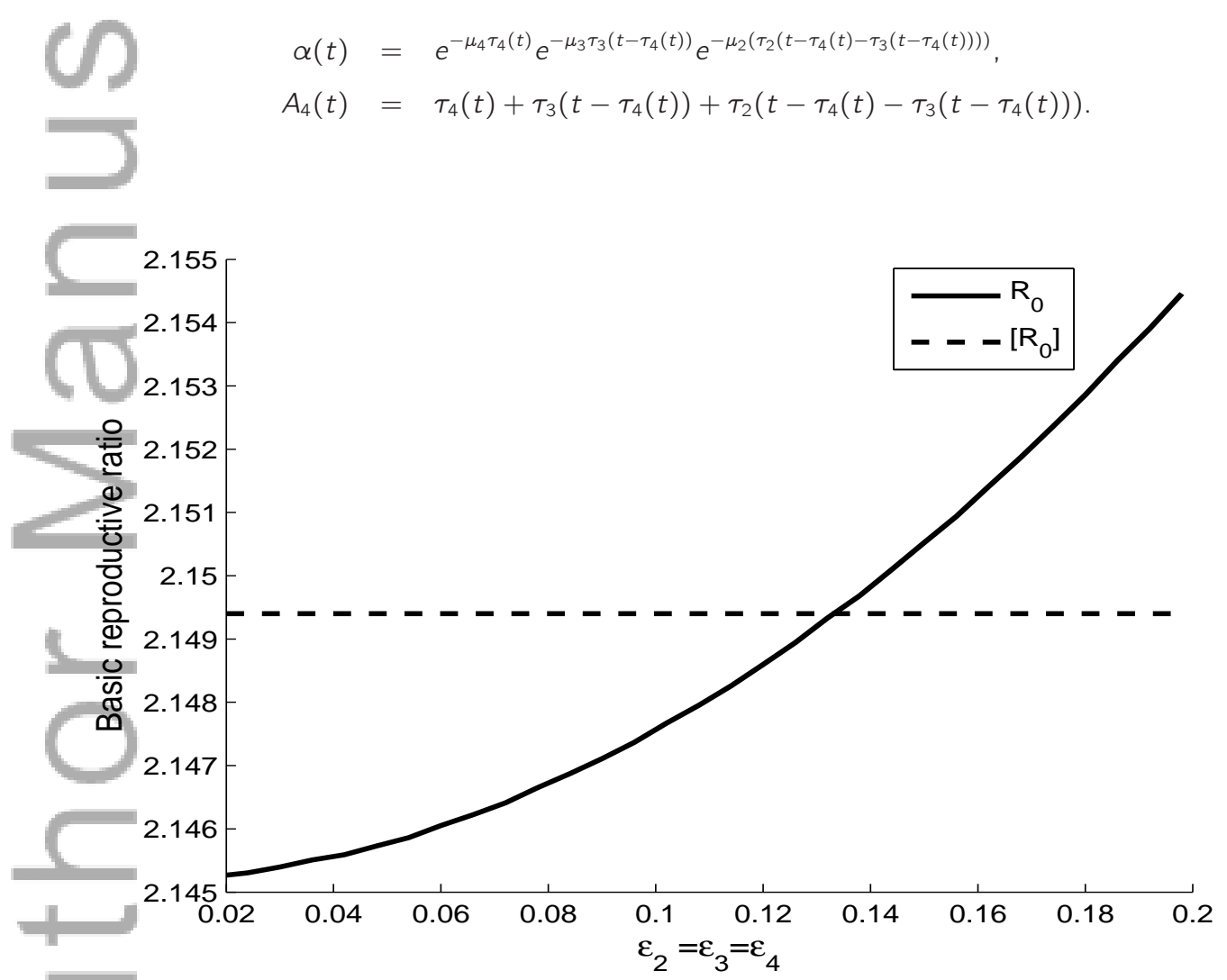

Figure 3. The graph of $\mathcal{R}_{0}$ (solid line) and time-averaged $\left[\mathcal{R}_{0}\right]$ (dashed line) versus amplitudes $\epsilon_{2}\left(=\epsilon_{3}=\epsilon_{4}\right) \in[0.02,0.2]$. Baseline parameter values: $\tau_{20}=5$, $\tau_{30}=10, \tau_{40}=2, \phi_{2}=\phi_{3}=\phi_{4}=270, \delta=0.5, p=200 / 365, \mu_{2}=0.01, \mu_{3}=\mu_{4}=0.025, \mu_{1}=0.09, \omega=365$.

Figures 3 and 4 report the simulations involving multiple periodic delays. Figure 3 shows that the increase of the amplitude $\epsilon_{2}, \epsilon_{3}$ and $\epsilon_{4}$ simultaneously can change the basic reproductive ratio $\mathcal{R}_{0}$ of system (47) from below the time-averaged basic reproductive ratio $\left[\mathcal{R}_{0}\right]$ to above. Hence, using the time-averaged delay differential system can either underestimate or overestimate the basic reproductive ratio of the corresponding period system of DDEs.

In Figure 4, we change the phase $\phi_{3}$ over the interval $[0,730]$ while keeping $\tau_{2}(t), \tau_{4}(t)$ and $\epsilon_{3}$ unchanged. We notice the 1 -year periodicity of $\mathcal{R}_{0}$ as a function of $\phi_{3}$. Moreover, an increase of phase of $\phi_{2}$ yields the shift of $\mathcal{R}_{0}$ to the right. For instance, the dashed curve corresponding to $\phi_{2}=100$ is just translation 100 to the right of the solid curve of $\mathcal{R}_{0}$ which corresponds to $\phi_{2}=0$. Therefore, difference in peak timings of the multiple periodic delays can change the value of the basic reproductive ratio $\mathcal{R}_{0}$, hence influence the state of survival and extinction of the population. 


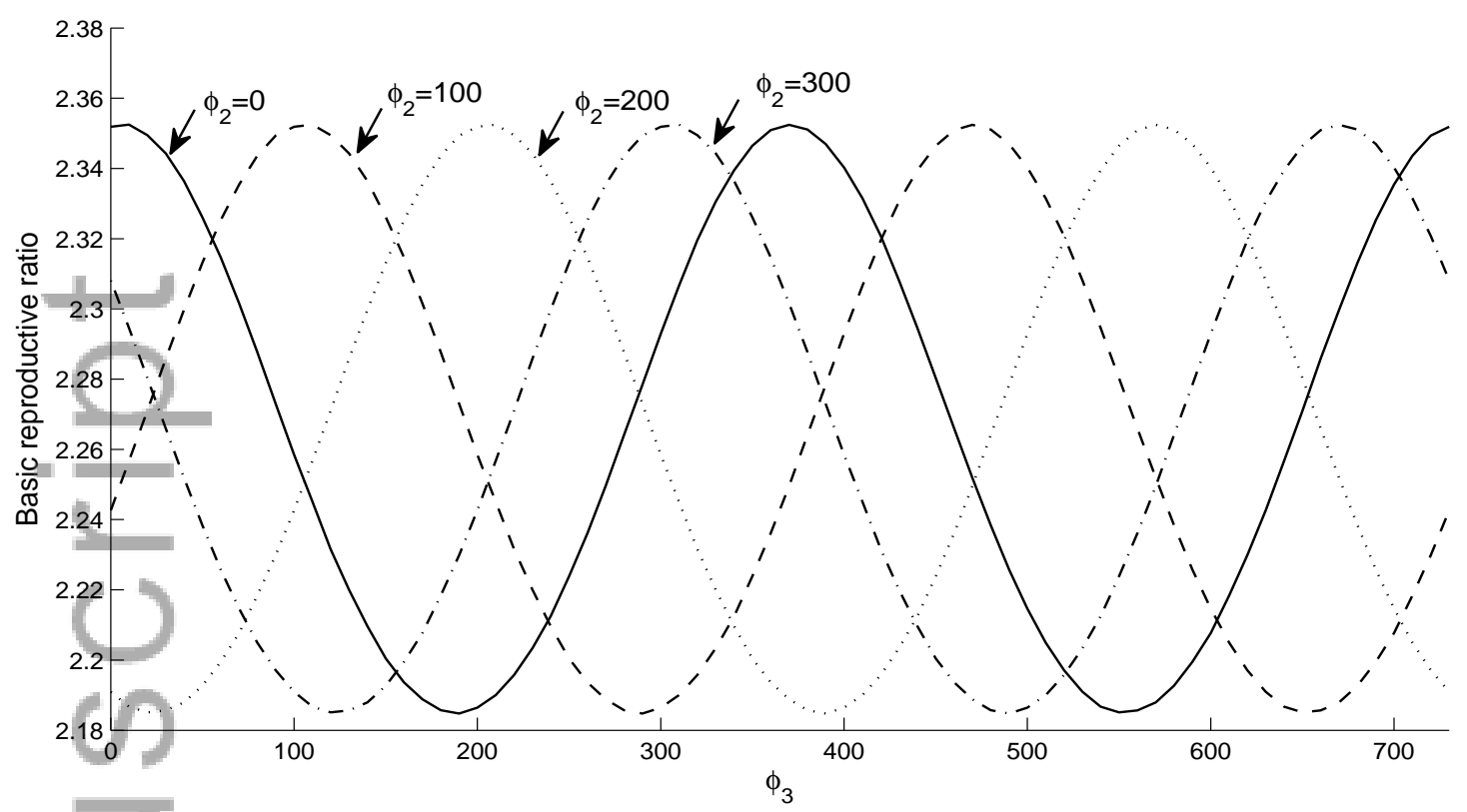

Figure 4 . The graph of basic reproductive ratio $\mathcal{R}_{0}$ versus $\phi_{3} \in[0,730]$. Baseline parameter values: $\tau_{20}=5, \epsilon=1, \tau_{30}=10, \epsilon_{3}=1, \tau_{40}=2, \epsilon_{4}=0$. Solid line represents $\mathcal{R}_{0}$ versus $\phi_{3}$ when $\phi_{2}=0$; Dashed line represents $\mathcal{R}_{0}$ versus $\phi_{3}$ when $\phi_{2}=100$; Dotted line represents $\mathcal{R}_{0}$ versus $\phi_{3}$ when $\phi_{2}=200$; Dash-dot line represents $\mathcal{R}_{0}$ versus $\phi_{3}$ when $\phi_{2}=300$.

\subsection{Comparison of $\mathcal{R}_{0}$ with the dominant Floquet multiplier}

Floquet multipliers are used in the theory of dynamical systems to determine the stability of periodic solutions. These can be calculated for DDE systems with time-dependent delay by adapting the method described in Luzyanina and Engelborghs [19] and Engelborghs et al. [13]. This is done by discretizing the time integration operator over the period and calculating the eigenvalues of the resulting matrix. For non-autonomous systems such as DDEs with time-dependent delays, if the the dominant Floquet multiplier has magnitude larger than one then the periodic solution is unstable. If the magnitude is less than one then the periodic solution is stable.

We consider the constant zero solution in our examples as a periodic solution with the same period $\omega$ as the period of the coefficients and delay function. In this way the stability of the zero solution could be investigated using Floquet multipliers as well as $\mathcal{R}_{0}$. We expect that as a model parameter is varied, $\mathcal{R}_{0}$ and the magnitude of the dominant Floquet multiplier of a DDE system will cross one at the same parameter value. Numerical experiments are consistent with this expectation and the plots are shown in Figure 5 for Example 1, as well as in Figure 6 for Example 2.

\subsection{The impact of temperature variation on $\mathcal{R}_{0}$}

Now we apply our algorithm to calculate the basic reproductive ratio of Ixodes scapularis tick population composed of 12 stages under temperature varying environmental condition. There are seven temperature-dependent time delays $\tau_{i}(t)$ $(i=2,4,6,7,9,10,12)$ and all others as constants. We take parameter values suggested in Ogden et al. [23]. Rodent abundance for immature ticks $R=200$, deer abundance for adults $D=20, \tau_{3}=21, \tau_{5}=3, \tau_{8}=5, \tau_{11}=10, \mu_{1}=0.005$, $\mu_{2}=0.002, \mu_{3}=0.006, \mu_{4}=0.006, \mu_{5}=0.65+0.049 \ln (1.01 / R), \mu_{6}=0.003, \mu_{7}=0.006, \mu_{8}=0.55+0.049 \ln (1.01 / R)$ $\mu_{9}=0.002, \mu_{10}=0.006, \mu_{11}=0.5+0.049 \ln (1.01 / D), \mu_{12}=0.0001 .1971-2000$ normal temperature data are used for three weather stations Ontario, Canada: Port Stanley, Hanover and Wiarton Airport. In order to obtain the periodic time delays $\tau_{i}(t)(i=2,4,6,7,9,10,12)$, we firstly obtain the seven period temperature-dependent development rates, denoted by $d_{i}(t)$ $(i=2,4,6,7,9,10,12)$ using the methodology developed in Wu et al. [39] by utilizing the following formulaes given at each day of the year 


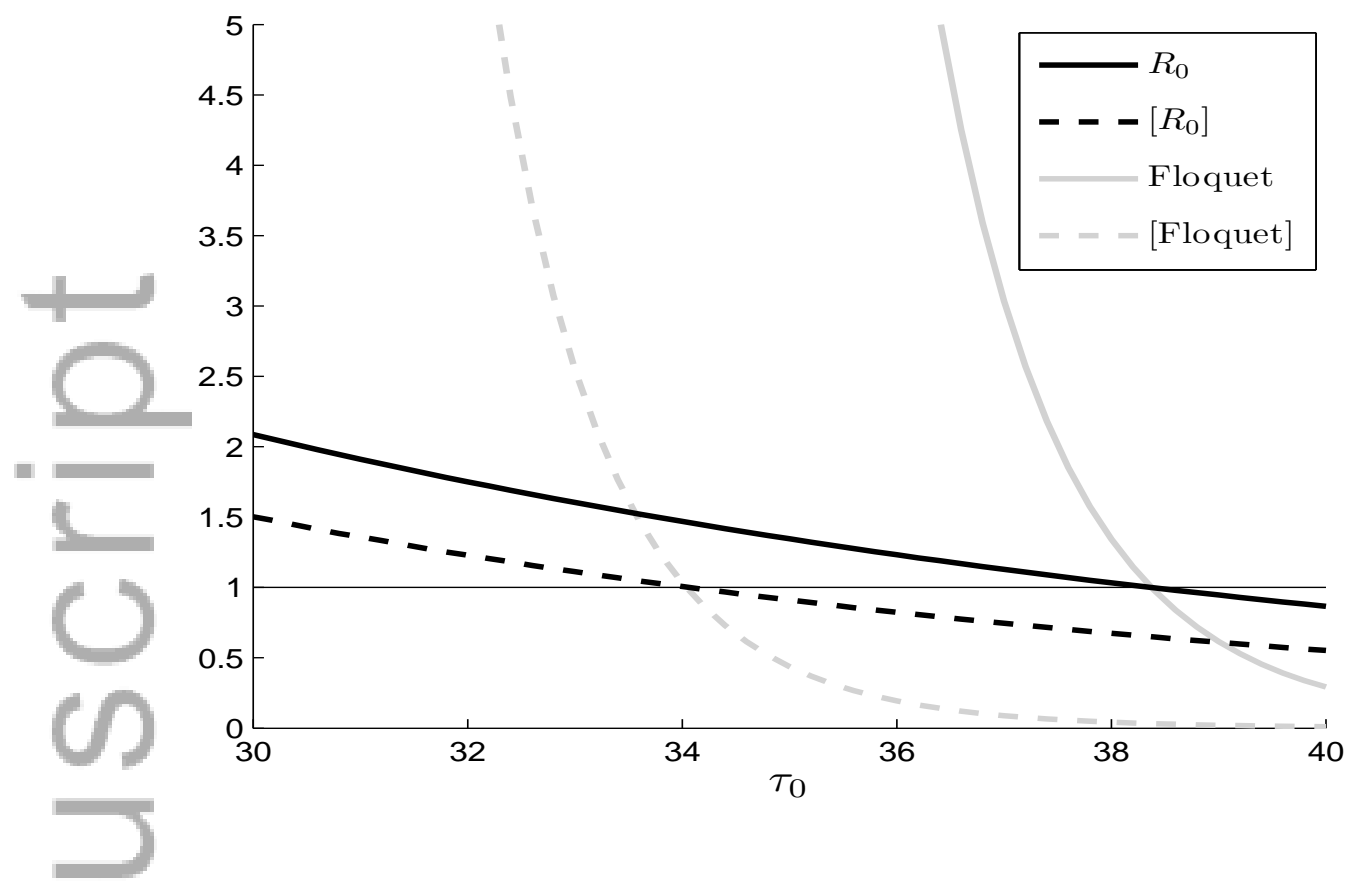

Figure 5. Plot of $\mathcal{R}_{0}$ (black) and the dominant Floquet Multiplier (grey) versus $\tau_{0} \in[30,40]$ for Example 1. Solid lines indicate results for periodic delay $(\varepsilon=0.5)$ and dashed lines are for constant delay $\left(\tau(t)=\tau_{0}\right)$. Baseline parameter values: $\phi=0, \gamma=0.1, d=0.133, p=8, \delta=0.5, \omega=365$.

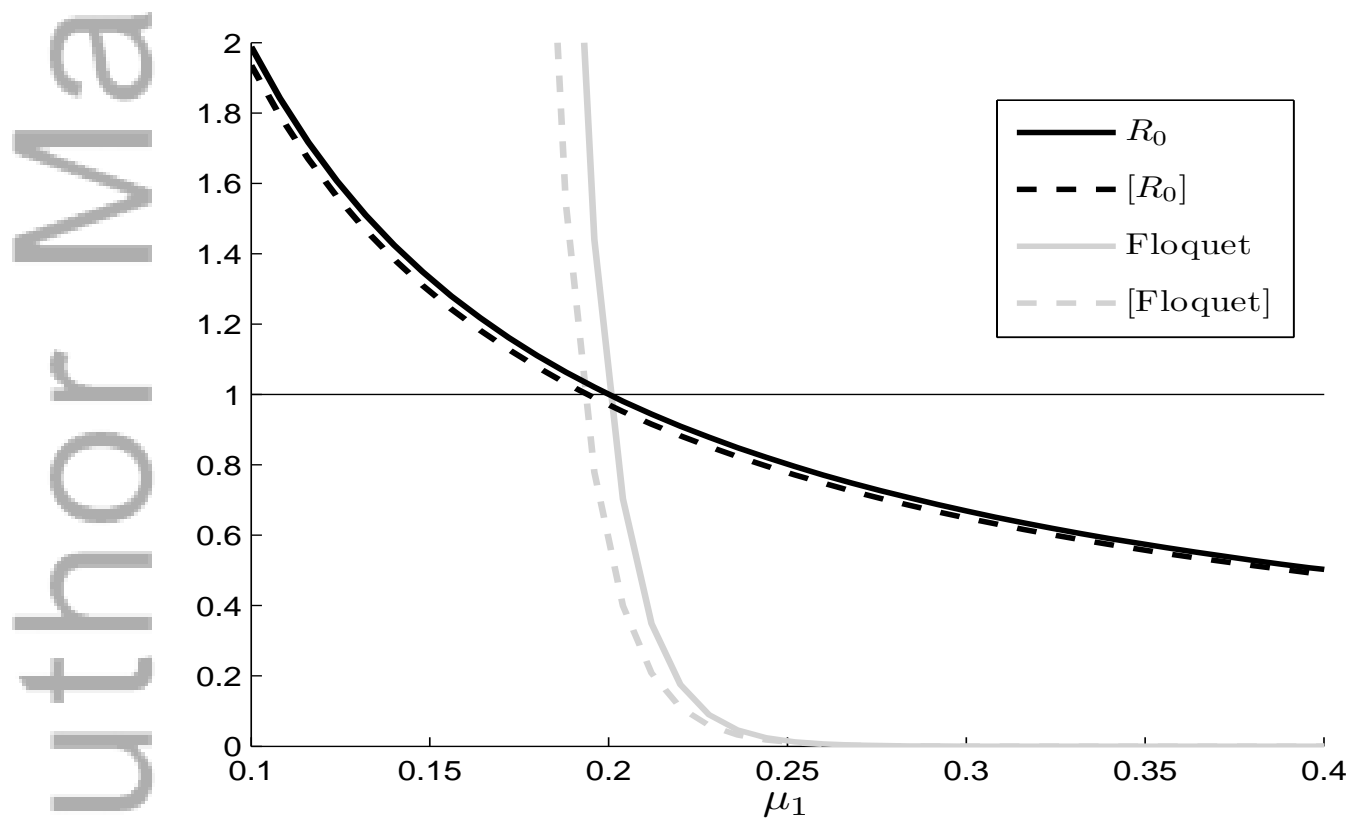

Figure 6. Plot of $\mathcal{R}_{0}$ (black) and the magnitude of the dominant Floquet multiplier (grey) versus $\mu_{1} \in[0.1,0.4]$. Solid lines indicate results for the periodic delay case $\left(\varepsilon_{2}=\varepsilon_{3}=\varepsilon_{4}=0.5\right)$ and dashed lines are for the constant delay case $\left(\tau_{i}(t)=\tau_{0 i}\right)$. Baseline parameter values: $\tau_{20}=5, \tau_{30}=10, \tau_{40}=2$, $\phi_{2}=\phi_{3}=\phi_{4}=270, \delta=0.5, p=200 / 365, \mu_{2}=0.01, \mu_{3}=\mu_{4}=0.025$ and $\omega=365$.

$$
\begin{aligned}
& 1 /\left(34234(T(t))^{-2.27}\right) \quad(\text { pre-eclosion period); } \\
& 0.0013 R^{0.515} \theta^{i}(T(t)) \quad \text { (time delay for host finding for larvae); } \\
& 1 /\left(101181(T(t))^{-2.55}\right) \quad \text { (larva-to-nymph); }
\end{aligned}
$$




$$
\begin{aligned}
& 0.0013 R^{0.515} \theta^{i}(T(t)) \quad \text { (time delay for host finding for nymphs); } \\
& 1 /\left(1596(T(t))^{-1.21}\right) \text { (nymph-to-adult); } \\
& 0.086 D^{0.515} \theta^{a}(T(t)) \quad \text { (time delay for host finding for adults); } \\
& 1 /\left(1300(T(t))^{-1.42}\right) \quad \text { (pre-oviposition period), }
\end{aligned}
$$

where $T(t)$ is temperature at time $t$ (unit ${ }^{\circ} \mathrm{C}$ ); $\theta^{i}(T(t))$ and $\theta^{a}(T(t))$ are temperature-dependent host activity proportions for immature and mature ticks (private communication). Note that the development rate of nymph-to-adult is affected by both temperature-dependent climate condition and temperature-independent diapause as mentioned in Ogden et al. [23], and more detail see the literature in $\mathrm{Wu}$ et al. [39]. Once the development rates are determined, the time-dependent delays $\left(\tau_{i}(t)\right)$ can be determined via backward calculation by the following relation

$$
\int_{t-\tau_{i}(t)}^{t} d_{i}(s) d s=1 .
$$

Therefore, the iterative time delays $A_{i}(t)$ are finally determined in terms of relation (9). The graphs of $\tau_{i}(t)$ and $A_{i}(t)$ are presented in Figure 7 and 8 respectively. Comparing to the ODE system of Ixodes scapularis tick population [39], the reproduction rate $p$ for the DDE model should significantly higher than that in ODE model. Setting

$$
p=3000 * \prod_{i=2}^{12} \frac{e^{\mu_{i} \bar{\tau}_{i}}}{1+\mu_{i} \bar{\tau}_{i}}
$$

where $\bar{\tau}_{i}$ is average time delay between successive stages, and using our developed algorithm, we estimate the basic reproductive ratio $\mathcal{R}_{0}$ to be $0.3371,1.6200$ and 2.8806 in Wiarton Airpot, Hanover and Port Stanley, respectively. This shows that increasing temperature conditions can shorten the development time between two successive stages and the time for finding hosts, and speed up maturation to egg-laying females, thereby increasing $\mathcal{R}_{0}$. Thus we can estimate the value of $\mathcal{R}_{0}$ subject to changing temperature conditions, and this has significant implications for the survival of the tick population.

\section{Discussion}

We derived a stage-structured population model to incorporate the variable development time in each stage of development (e.g., egg, larva, nymph and adult). The resulting model is a system of delay differential equations with periodic delay. Each component of the system represents a different life stage and the periodic delays represent the transition time between successive stages. Our model with $n=1$ (single stage) is consistent with the work of Schuhmacher and Thieme [31] in the special case with one exit maturation only. Let $r(t) \geq \epsilon>0$ be the development rate of a single stage, then there exists a unique $\tau_{i}(t)$ such that an individual must enter the stage at time $t-\tau_{i}(t)$ in order to reach maturation 1 and leave the stage at time $t$, i.e.,

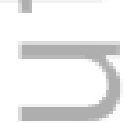

$$
\int_{t-\tau_{i}(t)}^{t} r(s) d s=1
$$

Differentiating the above equation with respect to time $t$ gives $r(t)-r\left(t-\tau_{i}(t)\right)\left(1-\tau_{i}^{\prime}(t)\right)=0$, hence we obtain $\frac{r(t)}{r\left(t-\tau_{i}(t)\right)}=$ $1-\tau_{i}^{\prime}(t)$. Our derived formula (17) within a single stage is exactly the same as Eqn (48) of [31]. However, we have developed a general model for the temperature-driven variable development of the parasite with multiple stages.

In the context of retarded functional differential equations (RFDEs), it is often difficult to analyse the asymptotic stability of either an equilibrium or a periodic solution due to the difficulty in dealing with the existence of infinitely many eigenvalues. There are intensive studies in the asymptotic stability of an equilibrium or a periodic solution for a linear autonomous/periodic RFDEs. To carry out numerical analysis of the eigenvalues, a commonly used approach is to reduce the infinite dimensional linear operator, as the solution operator for the linear autonomous RFDEs or monodromy operator in case of linear periodic RFDEs, to finite dimensional linear operator by means of pseudospectral collocation. Then the eigenvalues of the latter situation can be calculated by the standard methods for the associated matrix eigenvalues (see $[8,9]$ and references therein). In this paper, we 

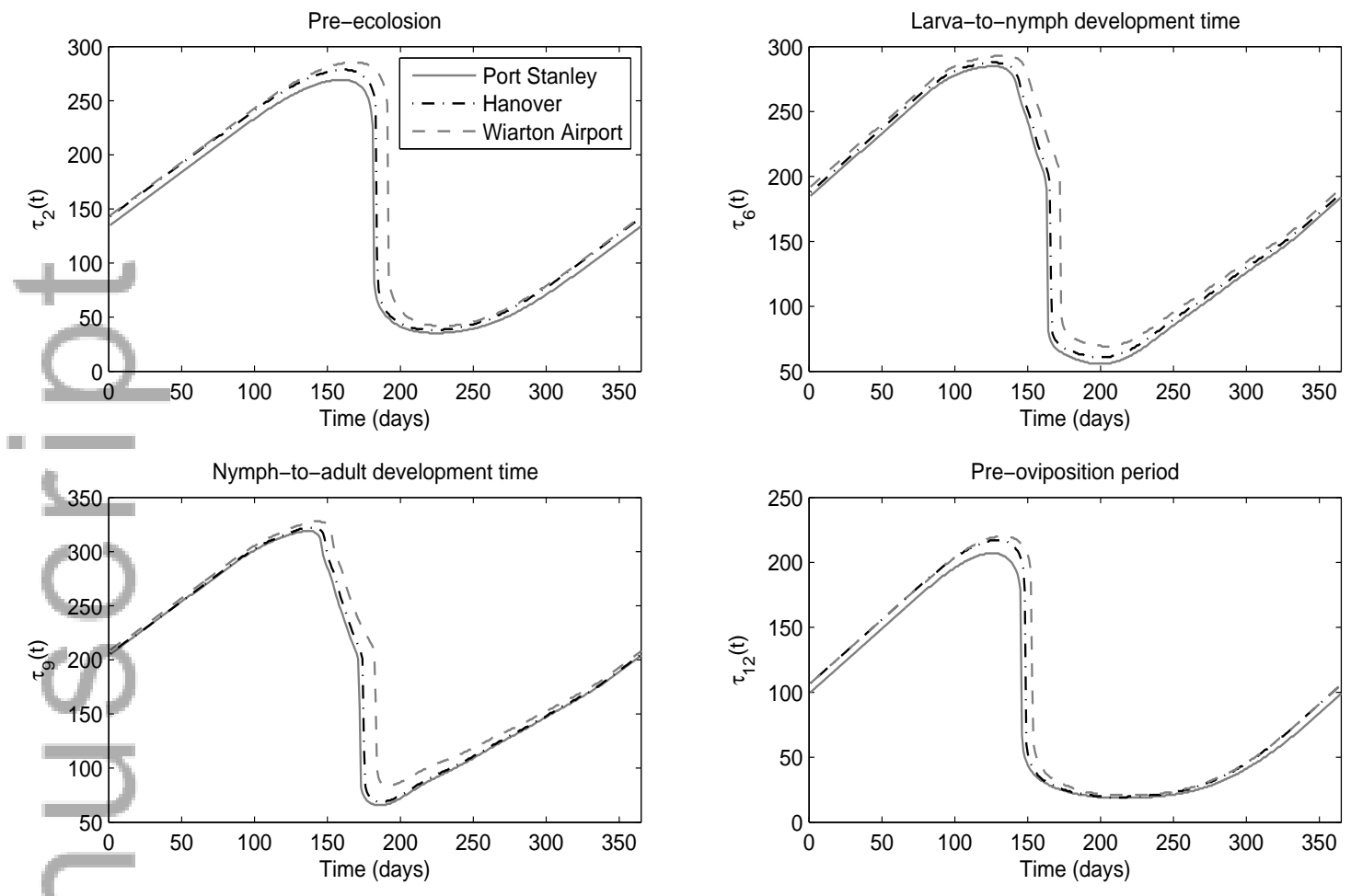

Figure 7. The graphs of interstadial development delay $\tau_{i}(t)(i=2,6,9,12)$ under different temperature scenarios. Vectors representing mean monthly 1971 - 2000 normal temperature in Port Stanley, Hanover and Wiarton Airport weather stations are given by [-5.5 -5.2 06.112 .417 .22019 .415 .6 9.44 .1 -2], [-7.1 -6.7-1.7 $5.41216 .919 .518 .514 .38 .32 .4-3.8$ ], [-6.8 -6.9 -2.2 4.710 .915 .618 .618 .1148 .42 .6 -3.3], respectively.

derived the basic reproductive ratio $\mathcal{R}_{0}$ for the scalar linearized periodic RFDE (linearized at the trivial solution) decoupled from the linearization of the full system at the population-extinction equilibrium. We have proposed a discretization-based method, where the periodic coefficients and the delays are both approximated by constants over a short time interval. This method then reduces the problem of calculating the spectral radius of a linear integral operator (defined as the basic reproductive ratio $\mathcal{R}_{0}$ ) to the calculation of the spectral radius of a finite dimensional matrix (the dominant eigenvalue of $\mathbf{X}$ ). Our numerical simulations indicate that this method is quite effective.

We compared our results with those from standard algorithms calculating the dominant Floquet multiplier [13, 19] in Figures 5 and 6 . Although the two algorithms both involved the discretization of an operator, the calculations were very different and it is encouraging to see that they are consistent in indicating which parameter regions the zero solution is stable or unstable. We would like to note that the computation time required to calculate $\mathcal{R}_{0}$ using our method depends on the form of the delay term (it depends on how $h^{-1}$ terms are calculated) whereas the calculation of Floquet multipliers is more straightforward. However, if we are interested in the stability of the zero solution as a parameter not involved in the delay term is varied (such as $\mu_{1}$ in Figure 6 ), then it is possible to compute $r_{i}, k_{i}, l_{i}$ as well as the $h^{-1}\left(t_{j}-k_{i} \omega\right)$ terms only once as the parameter is varied thereby saving computation time relative to the recalculating the Floquet multipliers each time.

We also performed simulations to gain insights on how the basic reproductive ratio $\mathcal{R}_{0}$ depends on the model parameters. With a single periodic delay, we noticed that the basic reproductive ratio $\mathcal{R}_{0}$ may increase as the amplitude of the periodic delay is increased (see Figure 1). We then focused on the issue of how amplitude/phase differences can influence the basic reproductive ratio $\mathcal{R}_{0}$ if multiple time-periodic delays are involved in a periodic system. We observed that time-averaged parameters should be avoided if seasonality is involved as this may lead to a bad estimate of the basic reproductive ratio and result in an inaccurate prediction of disease risk (see Figure 3). In particular, Figure 4 shows that the change of peak timings of two periodic delays can change the value of the basic reproductive ratio. The study in Ogden et al. [24] showed that seasonal activities of different tick instar changed due to the projected increased temperatures, from the current pattern "nymphal activities are ahead of larvae in 

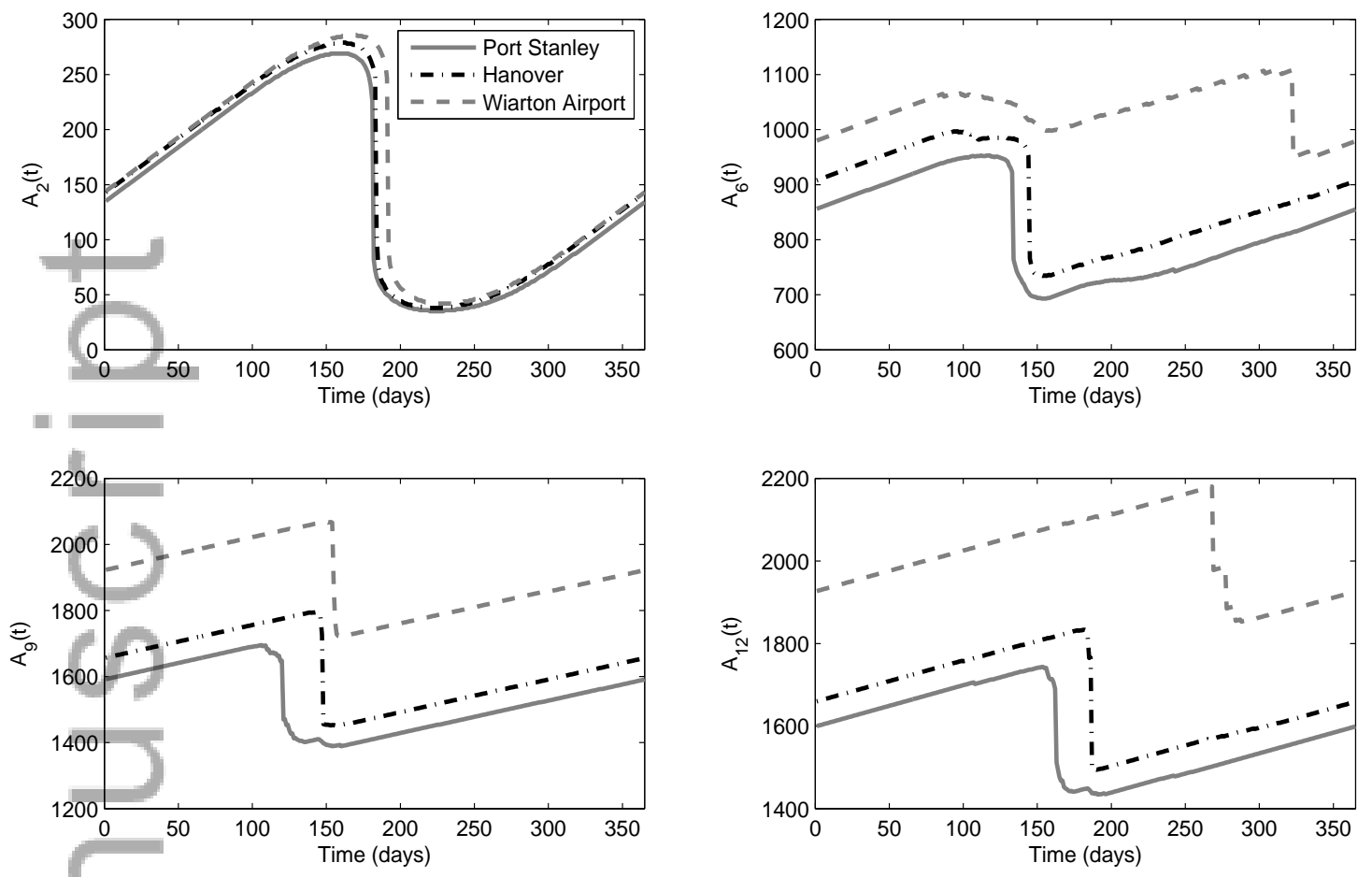

Figure 8. The graphs of time delay $A_{i}(t)(i=2,6,9,12)$ under different temperature scenarios. Vectors representing mean monthly $1971-2000$ normal temperature in Port Stanley, Hanover and Wiarton Airport weather stations are given by [-5.5 -5.2 06.112 .417 .22019 .415 .6 9.4 4.1 -2], [-7.1 -6.7 -1.7 5.4

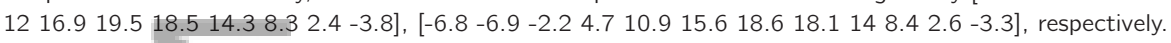

a year" to the one "larvae become active earlier than nymphs in a year" in the future. The result in [24] as well as our simulations indicate that switch of peak timings of larvae and nymphs may alter the basic reproductive ratio $\mathcal{R}_{0}$ for the tick population, and hence impact the estimate of tick population growth and associated tick-borne diseases such as Lyme disease.

\section{Acknowledgements}

The work was funded by the Natural Sciences and Engineering Research Council of Canada, and the Canada Research Chairs Program. JW would like to thank Professor Bacaër and Professor Thieme for bringing him the attention to a few critical references, and for related discussions.

\section{References}

1. R. M. Anderson and R. M. May, Infectious Diseases of Humans: Dynamics and Control, Oxford University Press, Oxford, 1991.

2. N. Bacaër and S. Guernaoui, The epidemic threshold of vector-borne diseases with seasonality, J. Math. Biol. 53 (2006) $421-436$.

3. N. Bacaër, Approximation of the basic reproduction number Ro for vector-borne diseases with a periodic vector population, Bull. Math. Biol. 69 (2007) 1067-1091.

4. N. Bacaër and R. Ouifki, Growth rate and basic reproduction number for populationmodels with a simple periodic factor, Math. Biosci. 210 (2007) 647-658.

5. N. Bacaër and X. Abdurahman, Resonance of the epidemic threshold in a periodic environment, J. Math. Biol. 57 (2008) 649-673.

6. N. Bacaër and M. G. M. Gomes, On the final size of epidemics with seasonality, Bull. Math. Biol. 71 (2009) 1954-1966.

7. N. Bacaër, A Short History of Mathematical Population Dynamics, Springer, London, 2011. 
8. D. Breda, S. Maset, and R. Vermiglio, Approximation of eigenvalues of evolution operators for linear retarded functional differential equations, SIAM J. Numer. Anal. 50 (2012) 1456-1483.

9. D. Breda, O. Diekmann, S. Maset and R. Vermiglio, A numerical approach for investigating the stability of equilibria for structured population models. J. Biol. Dyn. (available at http://dx.doi.org/10.1080/17513758.2013.78956).

10. T. Britton, Stochastic epidemic models: a survey, Math. Biosci.,225 (2010) 24-35.

11. O. Diekmann and J. A. P. Heesterbeek, Mathematical epidemiology of infectious disease:Model building, analysis and interpretation, Wiley, New York, 2000.

12. O. Diekmann, J. A. P. Heesterbeek and M. G. Roberts, The construction of next-generation matrices for compartmental epidemic models. J. R. Soc. Interface 7(47) (2010) 873-885.

13. K. Engelborghs, T. Luzyanina and D. Roose, Numerical bifurcation analysis of delay differential equations using DDE-BIFTOOL, ACM Trans. on Math. Software, 28 (2002) 1-21.

14. T. Gedeon, C. Bodelón and A. Kuenzi, Hantavirus transmission in sylvan and peridomestic environments, Bull. Math. Biol. 72 (2010) 541-564.

15. W. S. C. Gurney, S. P. Blythe and R. M. Nisbet, Nicholsons blowflies revisited, Nature 287 (1980) 17-21.

16. X. Hu, Threshhold dynamics for a Tuberculosis model with seasonality, Math. Biosci. Eng. 9 (2012) 111-122.

17. P. Jagers and O. Nerman, Branching processes in periodically varying environment, Ann. Prob. 13 (1985) 254-268.

18. Y. Lou, J. Wu and $\mathrm{X}$. Wu, Impact of climate warming and biodiversity on Lyme pathogen transmission in seasonal tick populations, submitted.

19. T. Luzyanina and K. Engelborghs, Computing Floquet multipliers for functional differential equations, Int. J. Bif. Chaos, 12 (2002) 2977-2989.

20. J. A. J. Metz and O. Diekmann, The dynamics of physiologically structured population. Lect. Notes. Biom. 68. Heidelberg: Springer, 1986.

21. R. M. Nisbet and W. S. C. Gurney, The systematic formulation of population models for insects with dynamically varying instar duration, Theor. Popul. Biol. 23 (1983) 114-135.

22. N. H. Ogden, L. R. Lindsay, G. Beauchamp, D. Charron, A. Maarouf, C. J. O'Callaghan, D. Waltner-Toews and I. K. Barker, Investigation of relationships between temperature and developmental rates of tick Ixodes scapularis (Acari: Ixodidae) in the laboratory and field, J. Med. Entomol. 41 (2004) 622-633.

23. N. H. Ogden, M. Bigras-Poulin, C. J. O'Callaghan, I. K. Barker, L. R. Lindsay, A. Maarouf, K. E. Smoyer-Tomic, D. Waltner-Toews and $\mathrm{D}$. Charron, A dynamic population model to investigate effects of climate on geographic range and seasonality of the tick Ixodes scapularis, Int. J. Parasitol. 35 (2005) 375-389.

24. N. H. Ogden, A. Maarouf, I. K. Barker, M. Bigras-Poulin, L. R. Lindsay, M. G. Morshed, C. J. O'Callaghan, F. Ramay, D. WaltnerToews and F. F. Charron, Climate change and the potential for range expansion of the Lyme disease vector Ixodes scapularis in Canada, Int. J. Parasitol. 36 (2006) 63-70.

25. N. H. Ogden, M. Radojević, X. Wu, V. R. Duvvuri, P. Leighton and J. Wu, Estimated effects of projected climate change on the basic reproductive number of the Lyme disease vector Ixods scapularis, Environ. Health. Perspect., In press.

26. J. F. M. Al-Omaria and S. A. Gourley, Dynamics of a stage-structured population model incorporating a state-dependent maturation delay, Nonlinear Analysis: RealWorld Applications 6 (2005) 13-33.

27. M. Otero, H. G. Solari and N. Schweigmann, A stochastic population dynamics model for Aedes Aegypti: formulation and application to a city with temperature climate, Bull. Math. Biol. 68 (2006) 1945-1974.

28. P. E. Parham and E. Michael, Modelling the effects of weather and climate change on malaria transmission, Environ. Health Persp. 118 (2010) 620-626.

29. P. van den Driessche and J. Watmough, Reproduction numbers and sub-threshold endemic equilibria for compartmental models of disease transmission, Math. Biosci. 180 (2002), 29-48.

30. M. A. Safil, M. Imran and A. B. Gumel, Threshold dynamics of a of a non-autonomous SEIRS model with quarantine and isolation. Theory Biosci. 131 (2012) 19-30.

31. K. Schuhmacher and H. Thieme, Some theoretical and numerical aspects of modelling dispersion in the development of ectotherms, Comput. Math. Applic. 15 (1988) 565-594.

32. P. L. Shaffer, Prediction of variation in development period of insects and mites reared at constant temperatures, Forum Envir. Ent. 12 (1983) 1012-1019.

33. P. J. H. Sharpe, G. L. Curry, D. W. de Michelle and C. L. Cole, Distribution model of organism development times, J. Theor. Biol. 66 (1977) 21-38.

34. H. R. Thieme, Renewal theorems for linear periodic Volterra integral equations, J. Integr. Equ. 7 (1984) 253-277.

35. W. Wang and X.-Q. Zhao, Threshold dynamics for compartmental epidemic models in periodic environments, J. Dyn. Diff. Equat. 20 (2008) 699-717. 
36. A. Wangombe, M. Andersson and T. Britton, A stage-structured stochastic epidemic model for tick-born diseases, http://people.su.se/ tbrit/publ.html.

37. G. F. Webb, Theory of nonlinear age-dependent population dynamics, Marcel Dekker, New York, 1985.

38. X. Wu, V. R. Duvvuri and J. Wu, Modeling dynamical temperature influence on the Ixodes scapularis population, 2010 International Congress on Environmental Modelling and Software, 2010.

39. X. Wu, V. R. Duvvuri, Y. Lou, N. H. Ogden, Y. Pelcat and J. Wu, Developing a temperature-driven map of the basic reproductive numbers of the emerging tick vector of Lyme disease Ixodes scapularis in Canada. J. Theor. Biol. 319 (2013) 50-61.

40. P. Yan Distribution Theory, Stochastic Processes and Infectious Disease Modelling, In F. Brauer, P. van den Driessche and J. Wu, Lecture Notes in Mathematical Epidemiology, Springer, 2008.

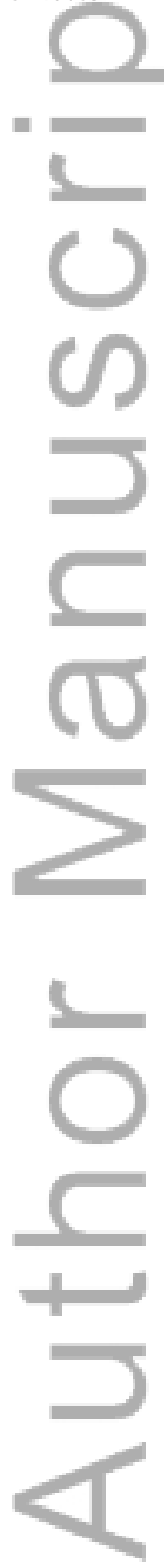




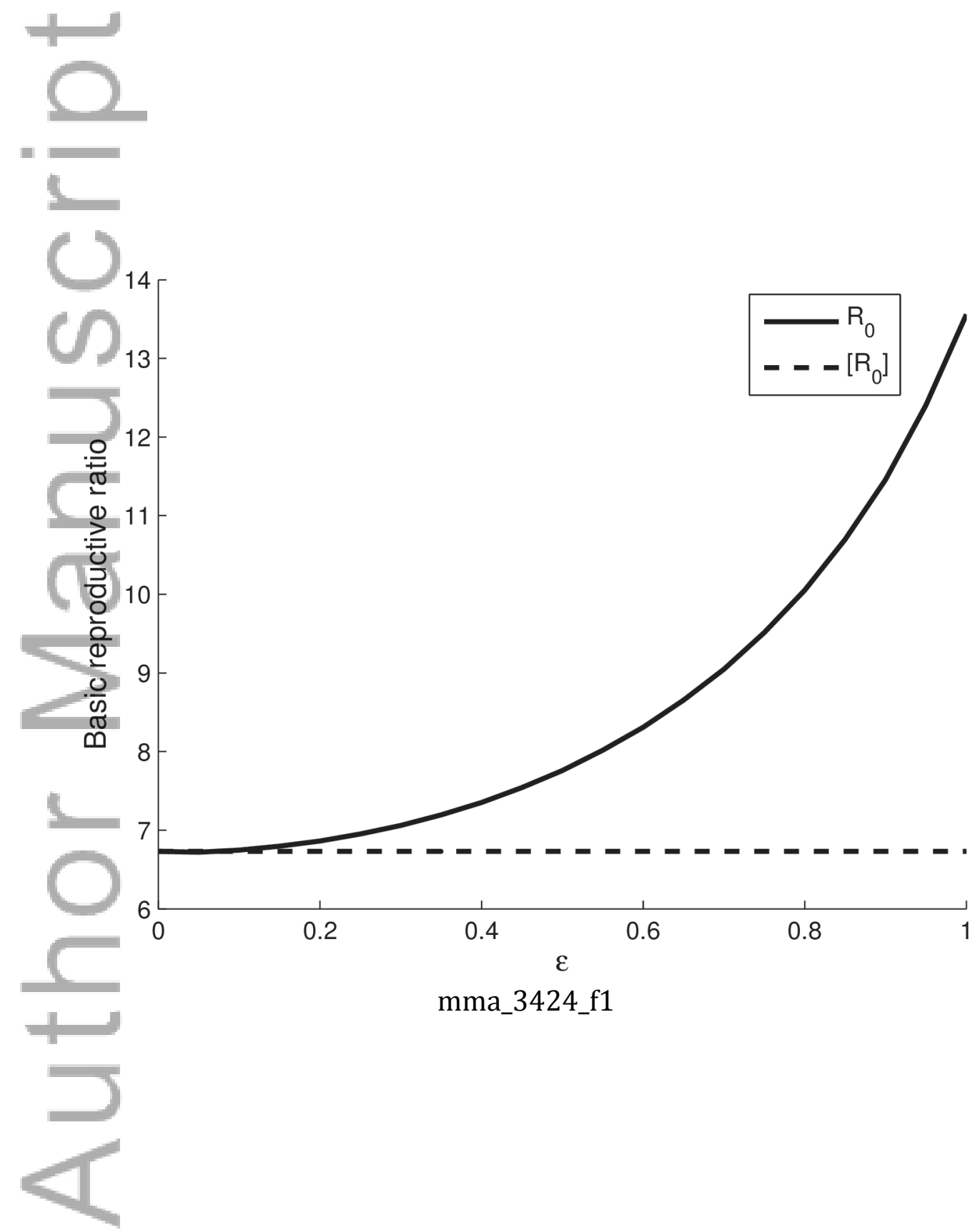

This article is protected by copyright. All rights reserved. 


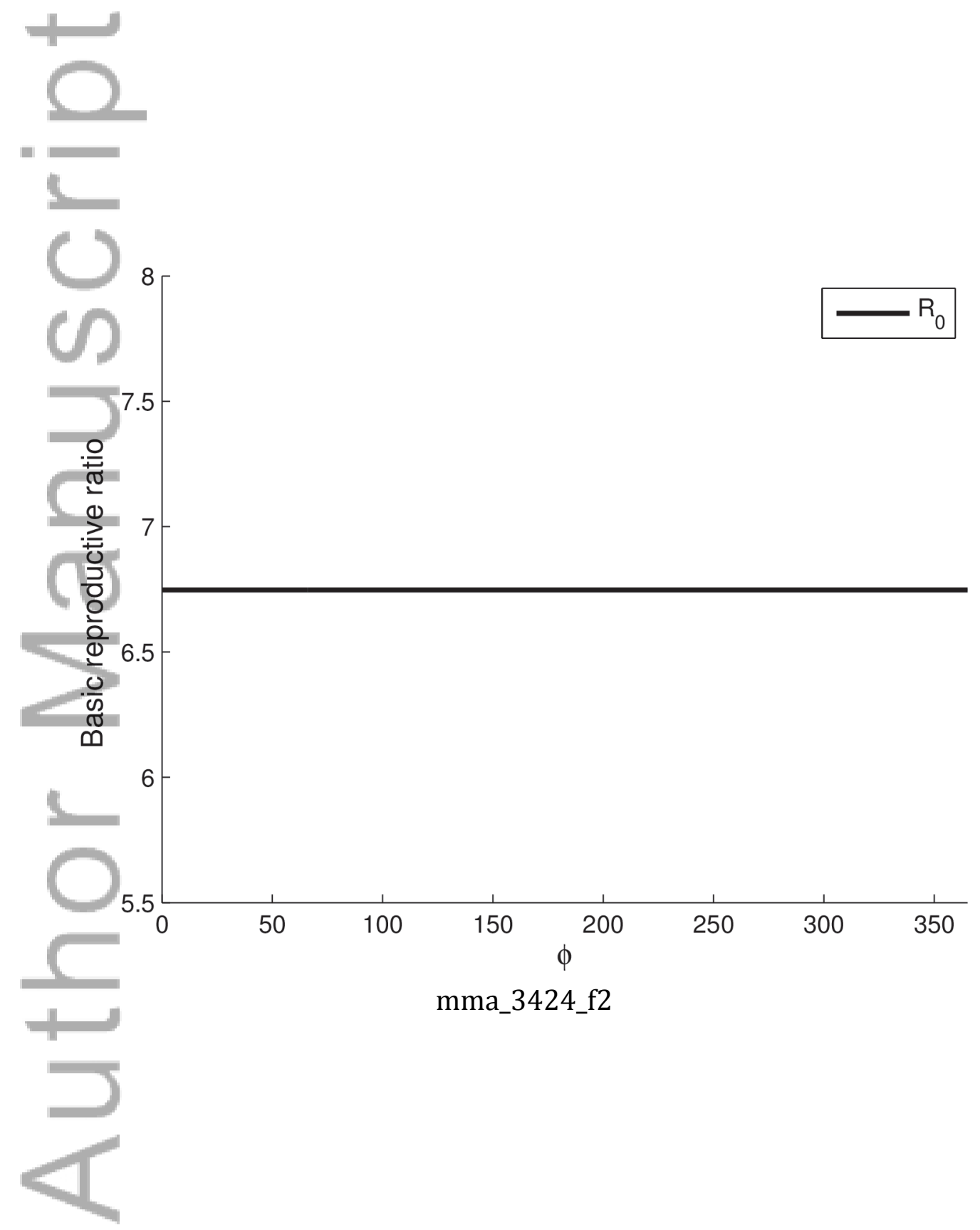

This article is protected by copyright. All rights reserved. 


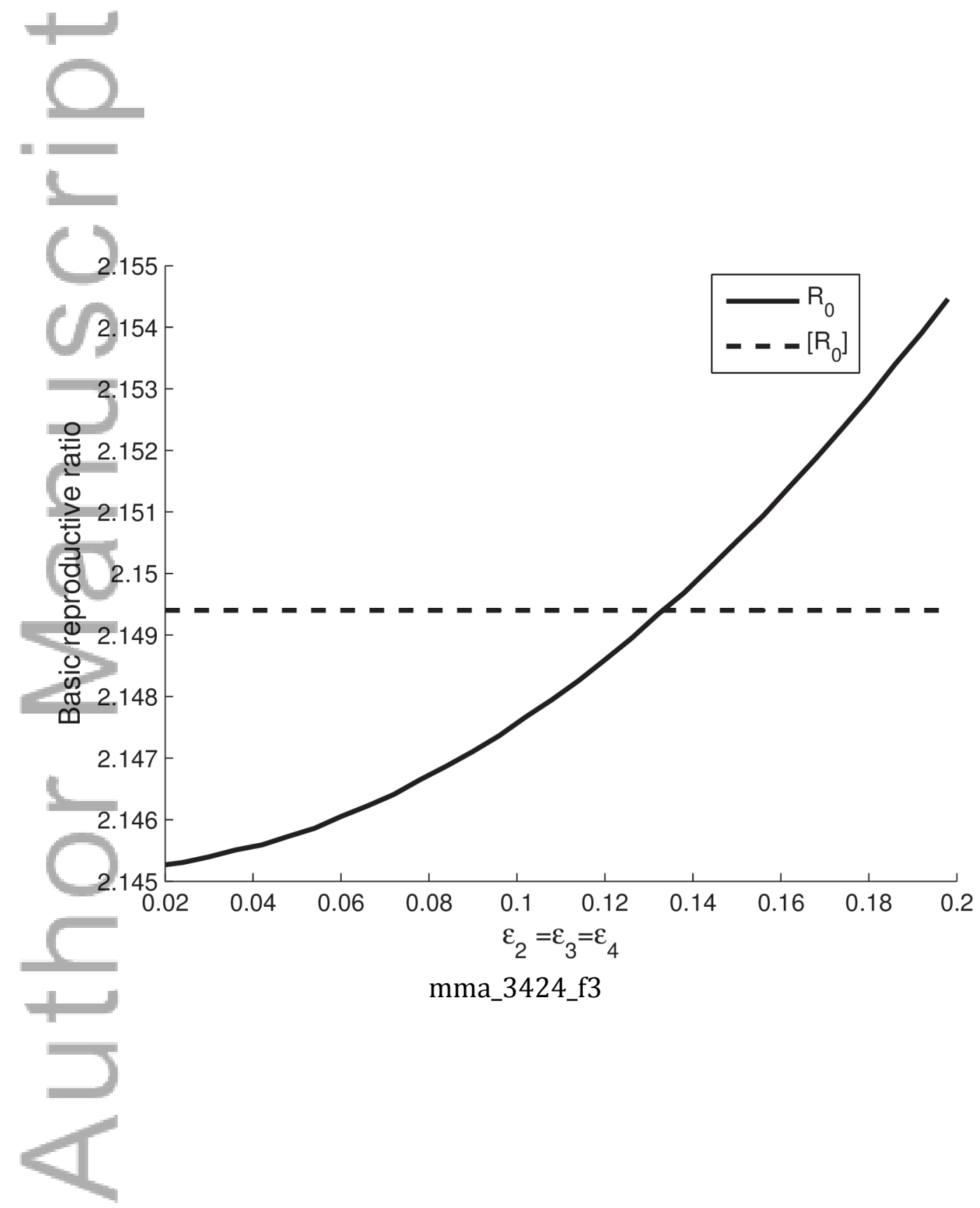

This article is protected by copyright. All rights reserved. 


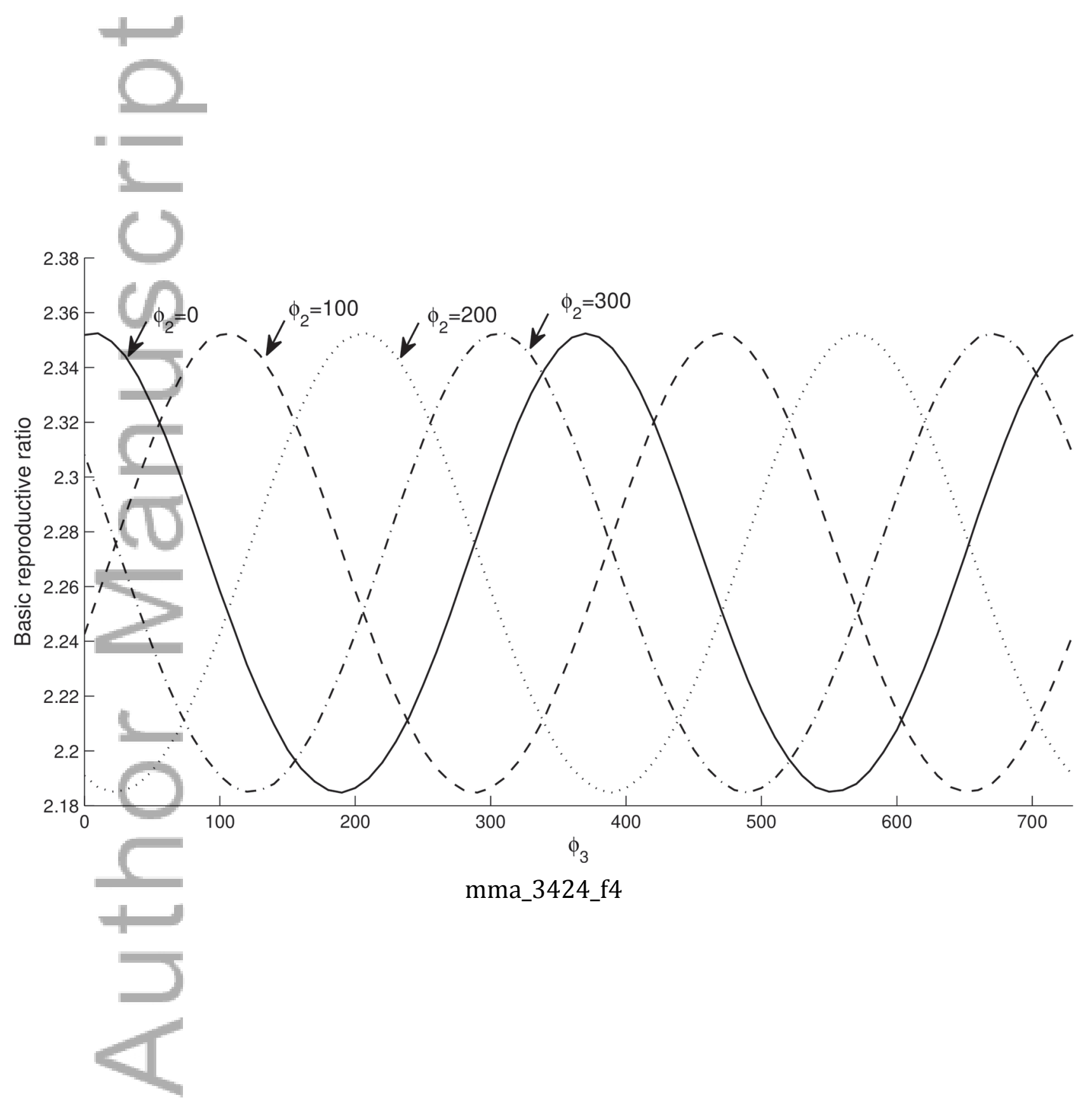

This article is protected by copyright. All rights reserved. 


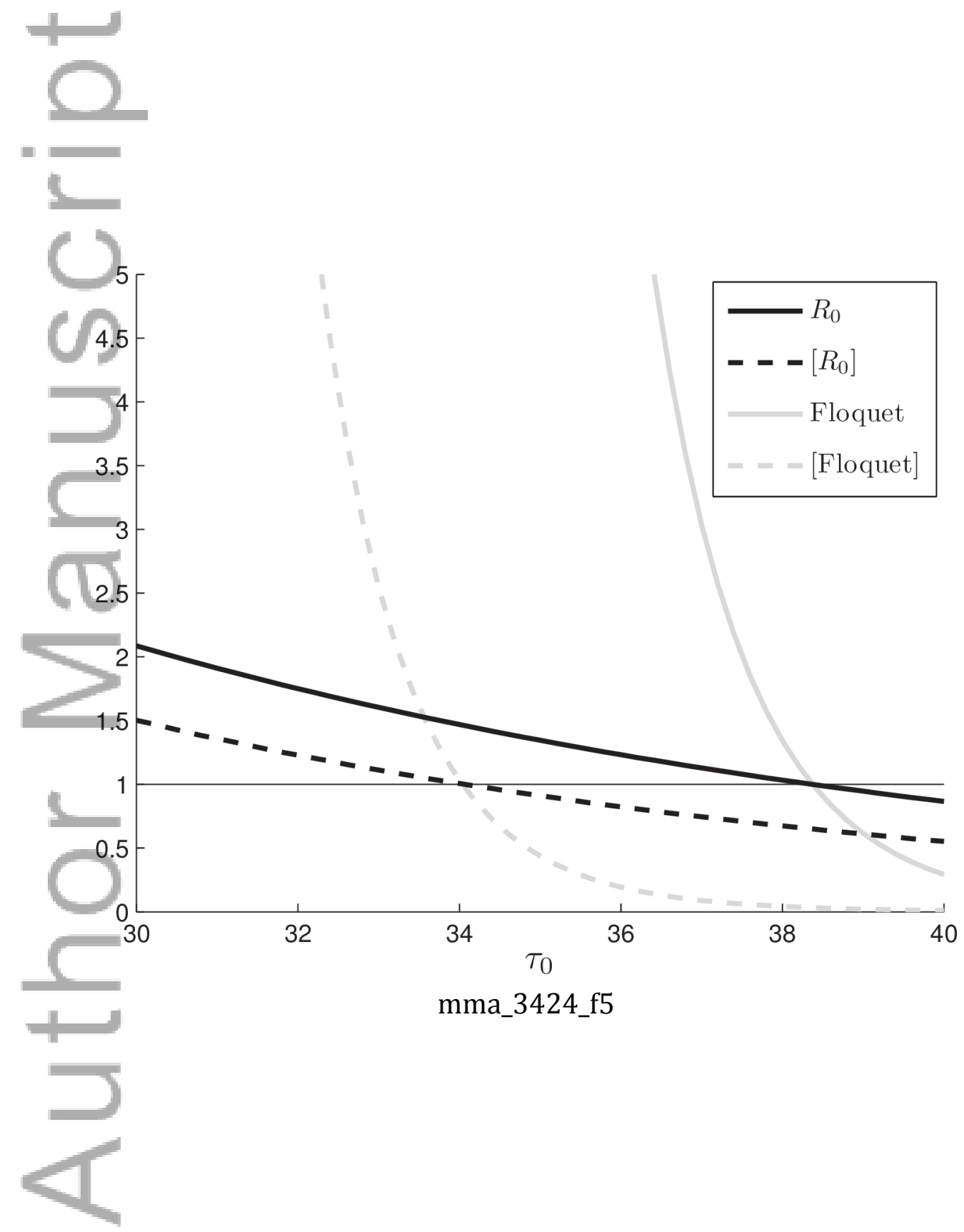

This article is protected by copyright. All rights reserved. 


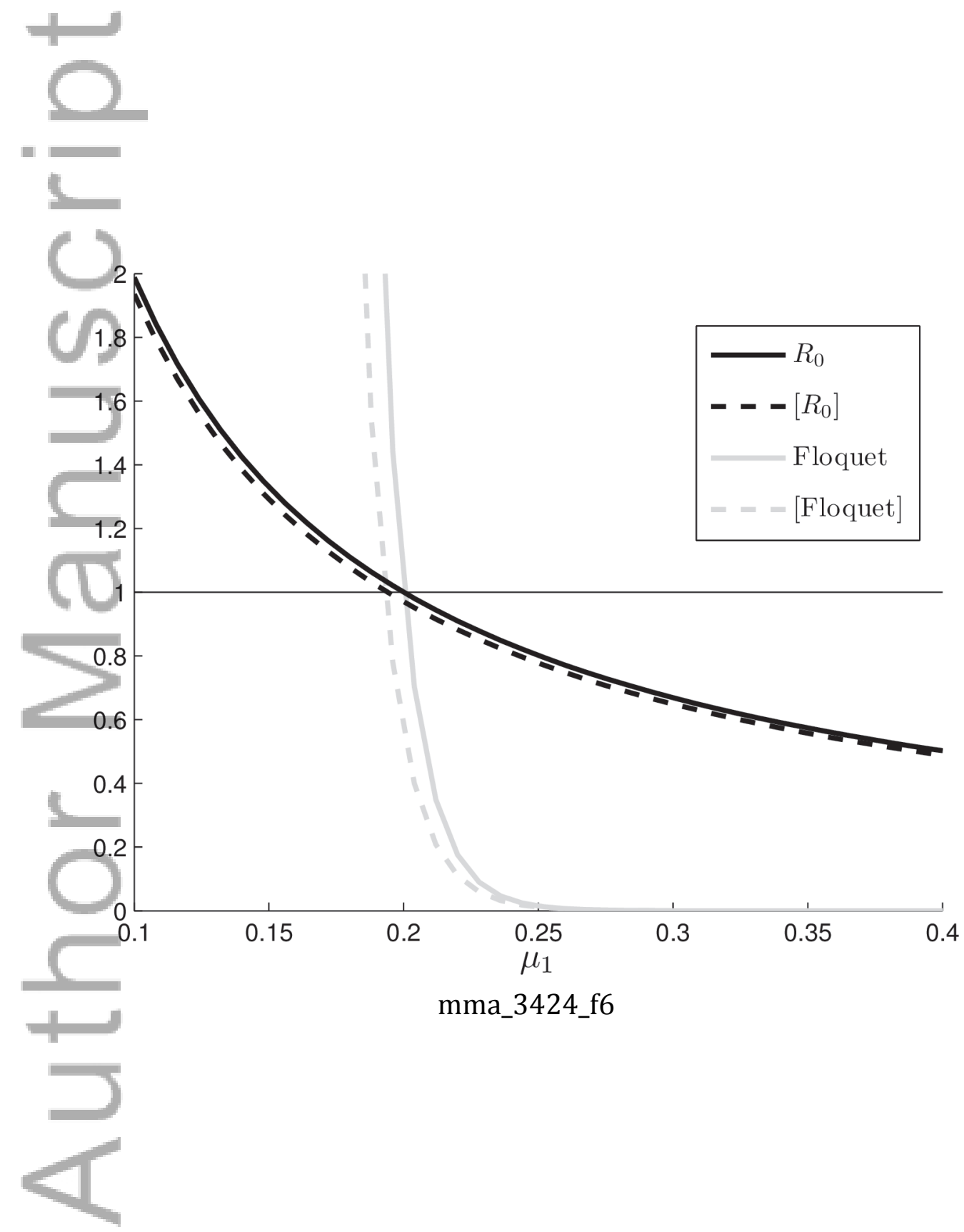

This article is protected by copyright. All rights reserved. 

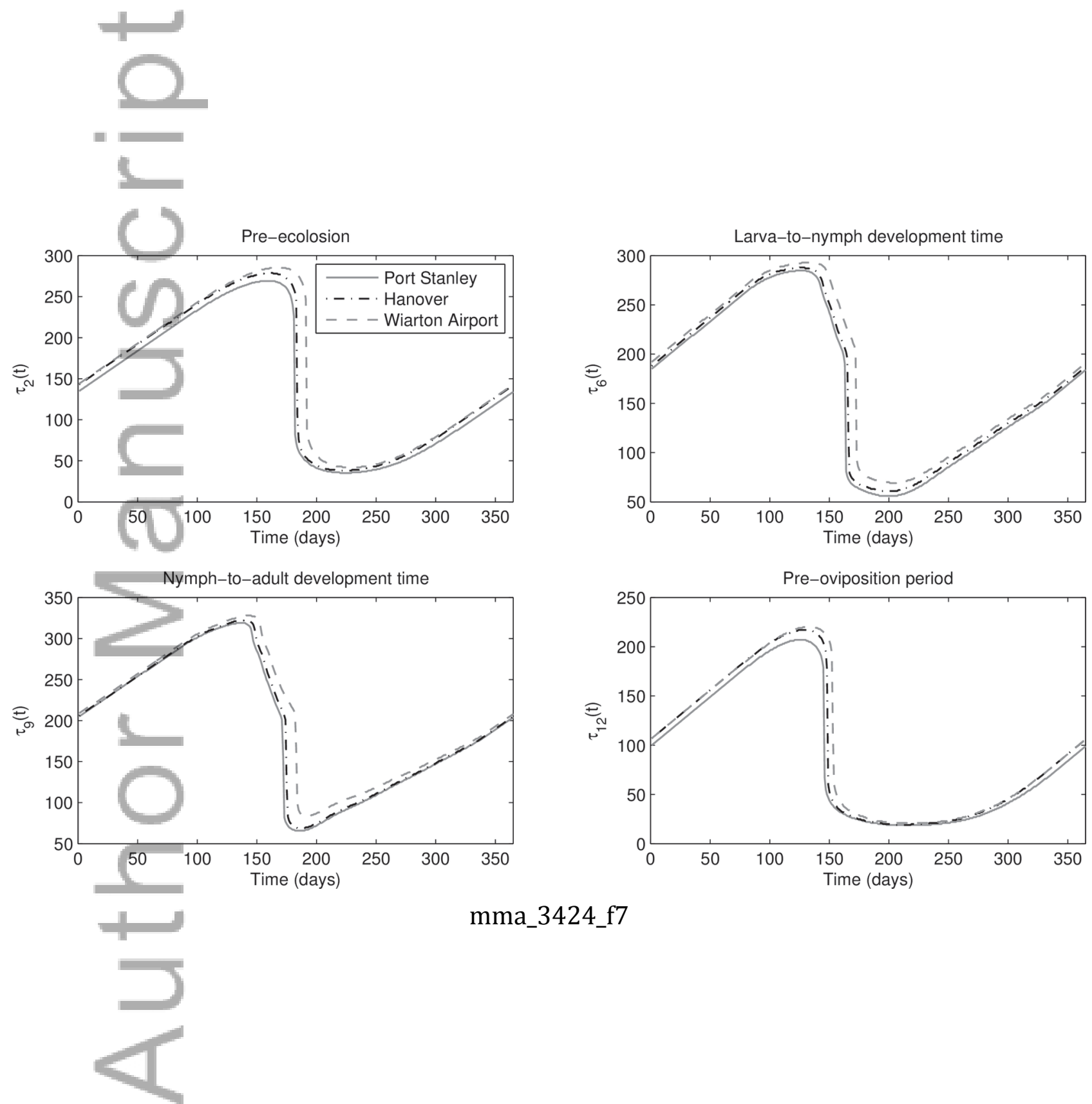

mma_3424_f7

This article is protected by copyright. All rights reserved. 

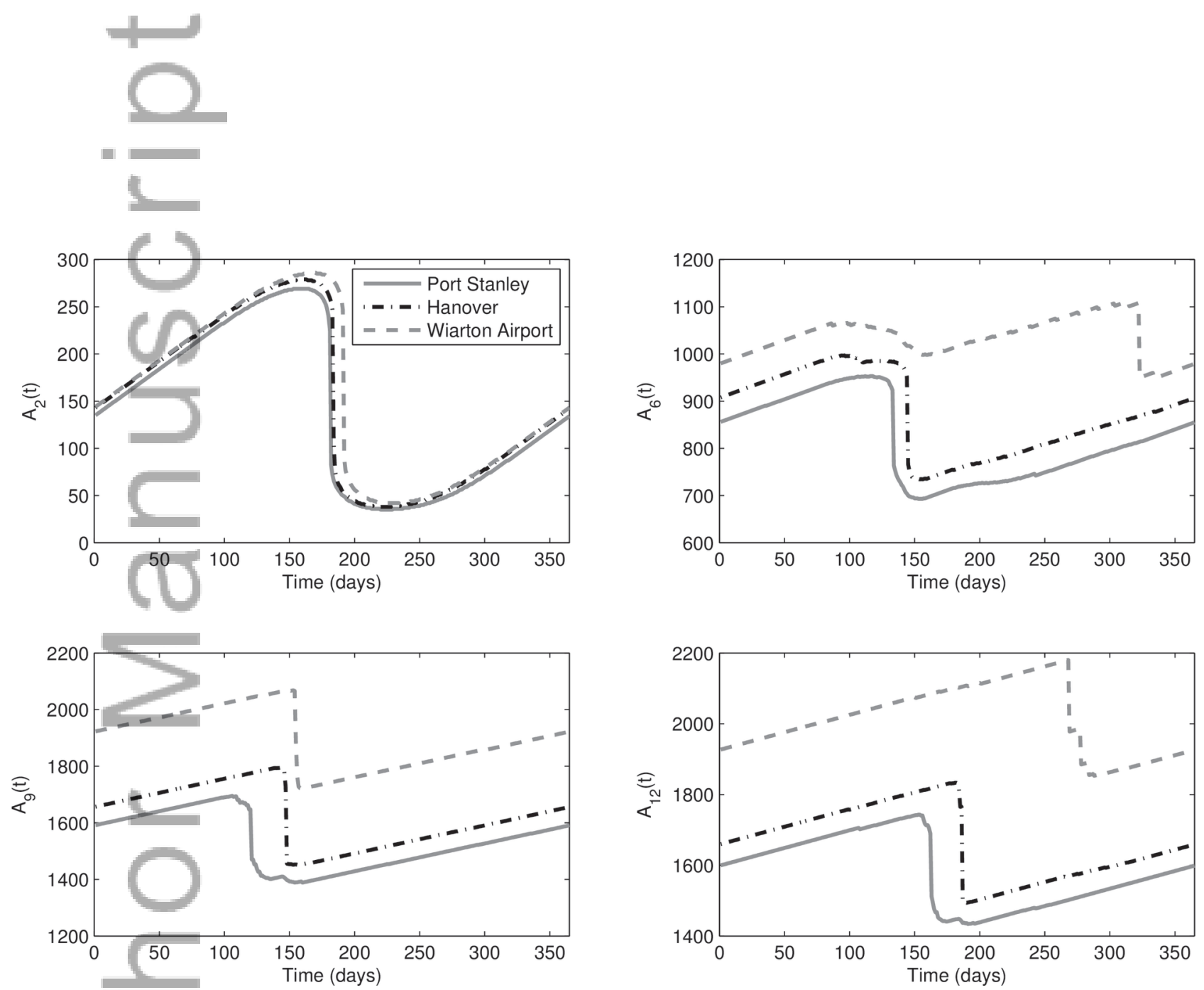

mma_3424_f8

This article is protected by copyright. All rights reserved. 